\title{
A TVD Uncertainty Quantification Method with Bounded Error Applied to Transonic Airfoil Flutter
}

\author{
Jeroen A. S. Witteveen* and Hester Bijl \\ Faculty of Aerospace Engineering, Delft University of Technology, Kluyverweg 1, \\ 2629HS Delft, The Netherlands.
}

Received 26 September 2008; Accepted (in revised version) 30 October 2008

Communicated by Jan S. Hesthaven

Available online 15 December 2008

\begin{abstract}
The Unsteady Adaptive Stochastic Finite Elements (UASFE) approach is a robust and efficient uncertainty quantification method for resolving the effect of random parameters in unsteady simulations. In this paper, it is shown that the underlying Adaptive Stochastic Finite Elements (ASFE) method for steady problems based on Newton-Cotes quadrature in simplex elements is extrema diminishing (ED). It is also shown that the method is total variation diminishing (TVD) for one random parameter and for multiple random parameters for first degree Newton-Cotes quadrature. It is proven that the interpolation of oscillatory samples at constant phase in the UASFE method for unsteady problems results in a bounded error as function of the phase for periodic responses and under certain conditions also in a bounded error in time. The two methods are applied to a steady transonic airfoil flow and a transonic airfoil flutter problem.
\end{abstract}

AMS subject classifications: 60H35, 65C30, 65N15, 65P99, 76M35

Key words: Total variation diminishing, extrema diminishing, error bounds, stochastic finite elements, uncertainty quantification, transonic flow, transonic flutter.

\section{Introduction}

Deterministic numerical solutions of engineering flow and fluid-structure interaction problems contain no information about the influence of parameter variations on the outputs of interest. Physical uncertainties are, however, present in practically all engineering applications due to, for example, varying atmospheric conditions, and production

*Corresponding author. Email addresses: j.a.s.witteveen@tudelft.nl (J. A. S. Witteveen), h.bijl@ tudelft.nl (H. Bijl) 
tolerances affecting material properties and the geometry. These inherent physical variations enter the computational problem through physical input parameters, and initial and boundary conditions. Especially, discontinuous solutions of shock waves in supersonic flow and bifurcation phenomena of aeroelastic systems are highly sensitive to this input variability. Dynamic fluid-structure interaction systems also amplify input variations with time.

Physical variability is here described in a probabilistic framework by random parameters with known probability density. The distribution functions and the statistical moments of outputs of interest are determined in order to obtain more reliable computational predictions, which can be utilized in robust design optimization and reducing design safety factors. In contrast, in structural reliability analysis input randomness is propagated to compute the probability of failure [4]. Failure probabilities are often small such that in that case the tails of the distribution are of interest.

The resulting mathematical formulation of the uncertainty quantification problem for output of interest $u(\mathbf{x}, t, \omega)$ is

$$
\mathcal{L}(\mathbf{x}, t, \omega ; u(\mathbf{x}, t, \omega))=\mathcal{S}(\mathbf{x}, t, \omega),
$$

with appropriate initial and boundary conditions. Operator $\mathcal{L}$ and source term $\mathcal{S}$ are defined on domain $D \times T \times \Omega$, where $\mathbf{x} \in D$ and $t \in T$ are the spatial and temporal dimensions with $D \subset \mathbb{R}^{d}, d=\{1,2,3\}$, and $T \subset \mathbb{R}$. The argument $\omega$ emphasizes that $u(\mathbf{x}, t, \omega)$ is a random event with the set of outcomes $\Omega$ of the probability space $(\Omega, \mathcal{F}, P)$ with $\mathcal{F} \subset 2^{\Omega}$ the $\sigma$-algebra of events and $P$ a probability measure. The probability space originates from $n_{\mathrm{a}}$ uncorrelated second order random parameters

$$
\mathbf{a}(\omega)=\left\{a_{1}(\omega), \cdots, a_{n_{\mathrm{a}}}(\omega)\right\} \in A,
$$

with probability density $f_{\mathbf{a}}(\mathbf{a})$ in Eq. (1.1) and its initial and boundary conditions, with parameter space $A \subset \mathbb{R}^{n_{a}}$.

For a single realization $\omega=\omega_{k}, u\left(\mathbf{x}, t, \omega_{k}\right)$ reduces to the deterministic function $u_{k}(\mathbf{x}, t)$ in terms of the spatial coordinates $\mathbf{x}$ and time $t$. The numerical approximation of $u_{k}(\mathbf{x}, t)$ can be obtained using standard spatial discretization methods and time marching schemes. A weighted approximation of the response surface $u^{*}(\mathbf{x}, t, \mathbf{a})$ based on $n_{\mathrm{s}}$ deterministic solutions $\left\{u_{k}(\mathbf{x}, t)\right\}_{k=1}^{n_{s}}$ is considered a solution of uncertainty quantification problem (1.1). Integration and sorting of $u^{*}(\mathbf{x}, t, \mathbf{a})$ results in the statistical moments $\mu_{\mathrm{u}_{i}}(\mathbf{x}, t)$

$$
\mu_{\mathrm{u}_{i}}(\mathbf{x}, t)=\int_{A} u^{*}(\mathbf{x}, t, \mathbf{a})^{i} f_{\mathbf{a}}(\mathbf{a}) \mathrm{d} \mathbf{a},
$$

and its probability distribution.

The classical approach of solving (1.1) by computing many deterministic solutions for randomly sampled parameter values in a Monte Carlo simulation [9] leads to impractically high computational costs for flow and fluid-structure simulations, which are already computationally intensive in the deterministic case. Non-intrusive Polynomial 
Chaos methods $[1,8,12,20,24,30]$ aim at reducing the number of deterministic solves by using a global polynomial interpolation of the samples in parameter space. An effective sampling in suitable Gauss quadrature points is employed in Stochastic Collocation approaches $[2,17,22,31]$. Current challenges in uncertainty quantification for computational fluid dynamics and fluid-structure interaction simulations include problems with discontinuities and unsteadiness [32].

Global polynomial approximations of discontinuities in probability space can result in oscillatory predictions and unphysical realizations. A more robust approximation is achieved by piecewise polynomial approximations of the response in adaptive finite elements discretizations of probability space $[6,15,16,23]$. These Adaptive Stochastic Finite Elements (ASFE) methods employ local Polynomial Chaos or Stochastic Collocation approximations in hypercube elements. An alternative Adaptive Stochastic Finite Elements formulation based on Newton-Cotes quadrature in simplex elements was recently also proposed [28]. Since the main motivation for performing uncertainty analysis is to obtain reliable computational predictions, it is important to assure the robustness of uncertainty quantification methods. In the deterministic finite volume community the total variation diminishing (TVD) and extrema diminishing (ED) properties [10,13] of finite volume methods ascertain that no unphysical solutions are predicted due to overshoots and undershoots near discontinuities. It is, therefore, useful to extend these concepts to uncertainty quantification methods in probability space.

In unsteady problems, uncertainty quantification methods usually require a fast increasing number of samples with time to maintain a constant accuracy. This effect is especially profound in problems with oscillatory solutions in which the frequency of the response is affected by the random parameters [19]. The random frequency results in increasing phase differences in the response, which consequently lead to an increasingly oscillatory response surface and more required samples. A Fourier Chaos basis can be a suitable alternative for approximating oscillatory responses [18]. Frequency domain methods have also been considered for solving linear stochastic operator equations [21].

Two Unsteady Adaptive Stochastic Finite Elements (UASFE) methods for oscillatory problems were proposed based on Newton-Cotes quadrature in simplex elements. The first approach is based on applying the uncertainty quantification interpolation to a timeindependent parameterization of oscillatory samples instead of to the unsteady samples themselves $[25,26]$. This results in a time-independent uncertainty quantification interpolation accuracy for the time-independent functionals. In the second method the oscillatory samples are scaled with their phase [27]. The uncertainty quantification interpolation of the samples is then performed at constant phase, which eliminates the effect of the increasing phase differences on the increase of the number of required samples. The latter method is not subject to a parameterization error, which improves the convergence behavior of the method, and it can resolve time-dependent functionals such as transient behavior. The formulation was also extended to multi-frequency responses of continuous structures by using a wavelet decomposition preprocessing step [29].

In this paper, it is shown that the Adaptive Stochastic Finite Elements method with 
Newton-Cotes quadrature in simplex elements is an extrema diminishing uncertainty quantification method in Section 2. It is also shown that the method is total variation diminishing for one random parameter and for multiple random parameters for first degree Newton-Cotes quadrature. It is proven in Section 3 that the Unsteady Adaptive Stochastic Finite Elements method with interpolation at constant phase results in a bounded error as function of the phase for periodic responses and under certain conditions also in a bounded error in time. The two methods are applied to a steady transonic airfoil flow and a transonic airfoil flutter problem in Section 4. The conclusions are summarized in Section 5.

\section{Adaptive Stochastic Finite Elements}

The Adaptive Stochastic Finite Elements method based on Newton-Cotes quadrature in simplex elements is presented in Section 2.1. It is shown under which conditions the approach is total variation diminishing in probability space in Section 2.2. In Section 2.3 it is proven that the method is extrema diminishing in probability space.

\subsection{Newton-Cotes quadrature in simplex elements}

Adaptive Stochastic Finite Elements with Newton-Cotes quadrature and simplex elements evaluate integral (1.2) by dividing parameter space $A$ in $n_{\mathrm{e}}$ non-overlapping simplex elements $A_{j}$

$$
\mu_{\mathrm{u}_{i}}(\mathbf{x}, t)=\sum_{j=1}^{n_{\mathrm{e}}} \int_{A_{j}} u^{*}(\mathbf{x}, t, \mathbf{a})^{i} f_{\mathbf{a}}(\mathbf{a}) \mathrm{d} \mathbf{a} .
$$

A piecewise polynomial approximation $w^{*}(\mathbf{x}, t, \mathbf{a})$ of the response $u^{*}(\mathbf{x}, t, \mathbf{a})$ is constructed based on $n_{\mathrm{s}}$ deterministic solutions $v_{j, k}(\mathbf{x}, t)=u^{*}\left(\mathbf{x}, t, \mathbf{a}_{j, k}\right)$ for the values of the random parameters $\mathbf{a}_{j, k}$ that correspond to the $\tilde{n}_{\mathrm{s}}$ Newton-Cotes quadrature points of degree $d$ in the element $A_{j}$

$$
\mu_{\mathrm{u}_{i}}(\mathbf{x}, t) \approx \mu_{\mathrm{w}_{i}}(\mathbf{x}, t)=\sum_{j=1}^{n_{\mathrm{e}}} \sum_{k=1}^{\tilde{n}_{\mathrm{s}}} c_{j, k} v_{j, k}(\mathbf{x}, t)^{i},
$$

where $c_{j, k}$ is the weighted integral of the Lagrange interpolation polynomial $L_{j, k}(\mathbf{a})$ through Newton-Cotes quadrature point $k$ in element $A_{j}$

$$
c_{j, k}=\int_{A_{j}} L_{j, k}(\mathbf{a}) f_{\mathbf{a}}(\mathbf{a}) \mathrm{d} \mathbf{a},
$$

for $j=1, \cdots, n_{\mathrm{e}}$ and $k=1, \cdots, \tilde{n}_{\mathrm{s}}$.

Here, first and second degree Newton-Cotes quadrature is considered, $d=1$ and $d=2$. The second degree Newton-Cotes formulation is combined with adaptive mesh refinement in probability space. The initial discretization of parameter space $A$ for the second degree adaptive scheme consists of the minimum of $n_{\mathrm{e}_{\mathrm{ini}}}=n_{\mathrm{a}}$ ! simplex elements and 


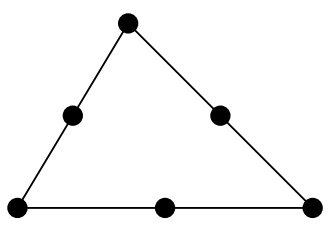

(a) Element

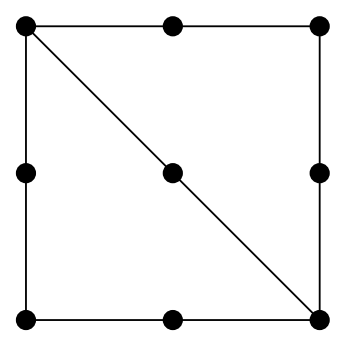

(b) Initial grid

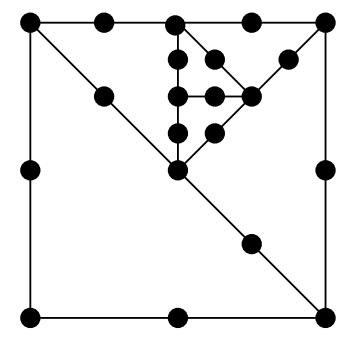

(c) Adapted grid

Figure 1: Discretization of two-dimensional parameter space $A$ using 2-simplex elements and second-degree Newton-Cotes quadrature points given by the dots.

$n_{\mathrm{sini}}=3^{n_{\mathrm{a}}}$ samples, see Fig. 1 . The example of Fig. 1 for two random input parameters can geometrically be extended to higher dimensional probability spaces. The elements $A_{j}$ are adaptively refined using a refinement measure $\rho_{j}$ based on the largest absolute eigenvalue of the Hessian $H_{j}$, as measure of the curvature of the response surface approximation in the elements, weighted by the probability $f_{j}$ contained by the elements

$$
f_{j}=\int_{A_{j}} f_{\mathbf{a}}(\mathbf{a}) \mathrm{d} \mathbf{a},
$$

with $\sum_{j=1}^{n_{\mathrm{e}}} f_{j}=1$. The stochastic grid refinement is terminated when $\delta_{n_{\mathrm{e}}}<\bar{\delta}$, where convergence measure $\delta_{n_{\mathrm{e}}}$ is defined as

$$
\delta_{n_{\mathrm{e}}}=\max \left(\frac{\left|\mu_{\mathfrak{u}_{\left\lfloor n_{\mathrm{e}} / 2\right\rfloor}}(\mathbf{x}, t)-\mu_{\mathrm{u}_{n_{\mathrm{e}}}}(\mathbf{x}, t)\right|_{\infty}}{\left|\mu_{\mathrm{u}_{n_{\mathrm{e}}}}(\mathbf{x}, t)\right|_{\infty}}, \frac{\left|\sigma_{\mathbf{u}_{\left\lfloor n_{\mathrm{e}} / 2\right\rfloor}}(\mathbf{x}, t)-\sigma_{\mathfrak{u}_{n_{\mathrm{e}}}}(\mathbf{x}, t)\right|_{\infty}}{\left|\sigma_{\mathfrak{u}_{n_{\mathrm{e}}}}(\mathbf{x}, t)\right|_{\infty}}\right),
$$

with $\mu_{\mathrm{u}}(\mathbf{x}, t)$ and $\sigma_{\mathrm{u}}(\mathbf{x}, t)$ the mean and standard deviation of $u(\mathbf{x}, t, \omega)$, or when a threshold for the maximum number of samples $\bar{n}_{\mathrm{s}}$ is reached. Convergence measure $\delta_{n_{\mathrm{e}}}$ can be extended to include higher statistical moments of the output.

Due to the location of the Newton-Cotes quadrature points the deterministic samples are reused in successive refinements and the samples are used in approximating the response in multiple elements. In elements where the quadratic second degree interpolation results in an extremum other than in a quadrature point, the element is subdivided into $\tilde{n}_{\mathrm{e}}=2^{n_{\mathrm{a}}}$ subelements with a linear first degree Newton-Cotes approximation of the response without performing additional deterministic solves.

As is common in multi-element methods, the probability of the random parameters $\mathbf{a}(\omega)$ is assumed to be zero outside a finite domain. Probability distributions on infinite domains are truncated at a small enough threshold value for the probability, such that the truncation error is small compared to other numerical errors that occur in practical applications. 


\subsection{Total variation diminishing}

It is shown that Adaptive Stochastic Finite Elements based on Newton-Cotes quadrature in simplex elements is total variation diminishing for one random parameter in Section 2.2.1. In Section 2.2.2 it is argued that the method is also total variation diminishing in higher dimensional probability spaces up to first degree Newton-Cotes quadrature.

\subsubsection{One-dimensional probability space}

Consider uncertainty quantification problem (1.1) with one random input parameter $a(\omega), n_{\mathrm{a}}=1$, on a bounded connected domain $a \in A$, with one-dimensional parameter space $A=[\min (a), \max (a)]$. Let response surface $u^{*}(\mathbf{x}, t, a)$ be a continuously differentiable function. The arguments $\mathbf{x}$ and $t$, and the index $*$ are omitted in the following for simplicity of the notation. Let sampling method $g$ result in a discrete set of $n_{\mathrm{s}}$ samples $\mathbf{v}=\left\{v_{1}, \cdots, v_{n_{\mathrm{s}}}\right\}=g(u(a))$ of response surface $u(a)$, with

$$
v_{k}=g_{k}(u(a))=u\left(a_{k}\right), \quad a_{k}=a\left(\omega_{k}\right), \quad k=1, \cdots, n_{\mathrm{s}},
$$

and

$$
a_{1} \leq a_{2} \leq \cdots \leq a_{n_{\mathrm{s}}}
$$

with $a_{1}=\min (a)$ and $a_{n_{\mathrm{s}}}=\max (a)$. Let interpolation method $h$ of the samples $\mathbf{v}$ result in a piecewise continuously differentiable interpolation function $w(a)=h(\mathbf{v})$ with $w\left(a_{k}\right)=$ $v_{k}$, which is continuously differentiable on subdomains $A_{j}$ of $A$ and continuous on the subdomain boundaries $\partial A_{j}$ with $j=1, \cdots, n_{\mathrm{e}}$. Let uncertainty quantification method $l$ evaluate (1.2) by approximating response surface $u(a)$ with interpolation

$$
w(a)=l(u(a))=h(g(u(a)))
$$

of the samples $\mathbf{v}$. Then the concepts total variation, total variation diminishing, and total variation conserving are defined in probability space as follows in correspondence to their definitions for finite volume methods in physical space in [10].

Definition 2.1. (Total variation) The total variation TV of response surface $u(a)$ in the space A of random parameter $a(\omega)$ is

$$
\operatorname{TV}(u)=\int_{A}\left|\frac{\partial u}{\partial a}\right| \mathrm{d} a
$$

The total variation of the continuous and piecewise continuously differentiable approximation $w(a)$ is

$$
\mathrm{TV}(w)=\sum_{j=1}^{n_{\mathrm{e}}} \operatorname{TV}\left(w_{j}\right)=\sum_{j=1}^{n_{\mathrm{e}}} \int_{A_{j}}\left|\frac{\partial w_{j}}{\partial a}\right| \mathrm{d} a .
$$

The total variation of the discrete set of samples $\mathbf{v}$ is

$$
\operatorname{TV}(\mathbf{v})=\sum_{k=1}^{n_{\mathrm{s}}-1}\left|v_{k+1}-v_{k}\right| .
$$


Definition 2.2. (Total variation diminishing) A set of samples $\mathbf{v}$ is total variation diminishing (TVD) with respect to response surface $u(a)$ if

$$
\operatorname{TV}(\mathbf{v}) \leq \mathrm{TV}(u) .
$$

Sampling method $g$ is TVD if the resulting set of samples $\mathbf{v}$ is TVD for all $u(a)$. Approximation $w(a)$ of response surface $u(a)$ is TVD if

$$
\operatorname{TV}(w) \leq \operatorname{TV}(u) .
$$

Uncertainty quantification method $l$ is TVD if the resulting approximation $w(a)$ is TVD for all $u(a)$.

Definition 2.3. (Total variation conserving) Interpolation $w(a)$ of samples $\mathbf{v}$ is total variation conserving (TVC) if

$$
\operatorname{TV}(w)=\mathrm{TV}(\mathbf{v}) .
$$

Interpolation method $h$ is TVC if the resulting interpolation $w(a)$ is TVC for all $\mathbf{v}$.

Based on these definitions it is proven below that Stochastic Finite Elements with Newton-Cotes quadrature in simplex elements is a TVD uncertainty quantification method for random parameter $a(\omega)$.

Lemma 2.1. Sampling method $g$ is TVD for random parameter $a(\omega)$.

Proof. For the total variation of the samples $\mathbf{v}=g(u(a))$ holds according to Definition 2.1

$$
\begin{aligned}
\operatorname{TV}(\mathbf{v}) & =\sum_{k=1}^{n_{\mathrm{s}}-1}\left|v_{k+1}-v_{k}\right|=\sum_{k=1}^{n_{\mathrm{s}}-1}\left|u\left(a_{k+1}\right)-u\left(a_{k}\right)\right| \\
& \leq \sum_{k=1}^{n_{\mathrm{s}}-1} \int_{a_{k}}^{a_{k+1}}\left|\frac{\partial u}{\partial a}\right| \mathrm{d} a=\int_{A}\left|\frac{\partial u}{\partial a}\right| \mathrm{d} a=\operatorname{TV}(u) .
\end{aligned}
$$

Since (2.13) holds for all $u(a)$, sampling method $g$ is TVD according to Definition 2.2.

Consider Stochastic Finite Elements uncertainty quantification method $l^{1}$ with first degree Newton-Cotes quadrature in simplex elements. Sampling method $g^{1}$ then results in $n_{\mathrm{s}}$ samples $\mathbf{v}^{1}$ in the vertices of the $n_{\mathrm{e}}$ simplex elements. Interpolation method $h^{1}$ results in a linear interpolation $w_{j}^{1}(a)$ of the samples $\mathbf{v}_{j}^{1}$ in the elements $A_{j}$. For one random parameter $a(\omega)$ sampling method $g^{1}$ results in $n_{\mathrm{s}}=n_{\mathrm{e}}+1$ samples $\mathbf{v}^{1}$. Interpolation method $h^{1}$ then results in the piecewise linear interpolation $w^{1}(a)$

$$
w^{1}(a)=w_{j}^{1}(a)=\frac{v_{j}^{1}\left(a_{j+1}-a\right)+v_{j+1}^{1}\left(a-a_{j}\right)}{a_{j+1}-a_{j}}, \quad \text { for } a \in A_{j}=\left[a_{j}, a_{j+1}\right], \quad j=1, \cdots, n_{\mathrm{e}} .
$$

Theorem 2.1. Uncertainty quantification method $l^{1}$ based on first degree Newton-Cotes quadrature in simplex elements is TVD for random parameter $a(\omega)$. 
Proof. The total variation of $w^{1}(a)$ is according to Definition 2.1 and (2.14)

$$
\begin{aligned}
\operatorname{TV}\left(w^{1}\right) & =\sum_{j=1}^{n_{\mathrm{e}}} \int_{A_{j}}\left|\frac{\partial w_{j}^{1}}{\partial a}\right| \mathrm{d} a=\sum_{j=1}^{n_{\mathrm{e}}}\left|\frac{v_{j+1}^{1}-v_{j}^{1}}{a_{j+1}-a_{j}}\right|\left(a_{j+1}-a_{j}\right) \\
& =\sum_{k=1}^{n_{\mathrm{s}}-1}\left|v_{k+1}^{1}-v_{k}^{1}\right|=\operatorname{TV}\left(\mathbf{v}^{1}\right) .
\end{aligned}
$$

Since (2.15) holds for all $\mathbf{v}^{1}$, interpolation method $h^{1}$ is TVC according to Definition 2.3. Lemma 2.1 gives

$$
\operatorname{TV}\left(w^{1}\right)=\operatorname{TV}\left(\mathbf{v}^{1}\right) \leq \operatorname{TV}(u)
$$

Since (2.16) holds for all $u(a)$, uncertainty quantification method $l^{1}$ is TVD according to Definition 2.2.

Consider Stochastic Finite Elements uncertainty quantification method $l^{2}$ with second degree Newton-Cotes quadrature in simplex elements. Sampling method $g^{2}$ then results in $n_{\mathrm{s}}$ samples $\mathbf{v}^{2}$ in the middle of the edges and in the vertices of the $n_{\mathrm{e}}$ simplex elements. Interpolation method $h^{2}$ results in a quadratic interpolation $w_{j}^{2}(a)$ of the samples $\mathbf{v}_{j}^{2}$ in the elements $A_{j}$. For one random parameter $a(\omega)$ sampling method $g^{2}$ results in $n_{\mathrm{s}}=2 n_{\mathrm{e}}+1$ samples. Interpolation method $h^{2}$ then results in quadratic approximation $w_{j}^{2}(a)$ in the element $A_{j}$ through the samples $v_{k}^{2}$ for $k=\{2 j-1,2 j, 2 j+1\}, j=1, \cdots, n_{\mathrm{e}}$. If the quadratic approximation $w_{j}^{2}(a)$ in an element $A_{j}$ has an extremum other than in a quadrature point $a_{k}$, i.e.,

$$
\min _{A_{j}}\left(w_{j}^{2}(a)\right)<\min \left(v_{2 j-1}^{2}, v_{2 j}^{2}, v_{2 j+1}^{2}\right) \vee \max _{A_{j}}\left(w_{j}^{2}(a)\right)>\max \left(v_{2 j-1}^{2}, v_{2 j}^{2}, v_{2 j+1}^{2}\right),
$$

then element $A_{j}$ is subdivided into $\tilde{n}_{\mathrm{e}}=2$ subelements with a linear first degree NewtonCotes approximation based on the samples $v_{k}$ with $k=\{2 j-1,2 j, 2 j+1\}$

$$
w_{j}^{2}(a)= \begin{cases}\frac{v_{2 j-1}^{2}\left(a_{2 j}-a\right)+v_{2 j}^{2}\left(a-a_{2 j-1}\right)}{a_{2 j}-a_{2 j-1}}, & a \in\left[a_{2 j-1}, a_{2 j}\right], \\ \frac{v_{2 j}^{2}\left(a_{2 j+1}-a\right)+v_{2 j+1}^{2}\left(a-a_{2 j}\right)}{a_{2 j+1}-a_{2 j}}, & a \in\left[a_{2 j}, a_{2 j+1}\right],\end{cases}
$$

Theorem 2.2. Uncertainty quantification method $l^{2}$ based on second degree Newton-Cotes quadrature in simplex elements is TVD for random parameter $a(\omega)$.

Proof. Two cases have to be considered to prove Theorem 2.2. In case (i) the quadratic approximation $w_{j}^{2}(a)$ in element $A_{j}$ has an extremum other than in a quadrature point $a_{k}$ (2.17)

$$
\min _{A_{j}}\left(w_{j}^{2}(a)\right)<\min \left(v_{2 j-1}^{2}, v_{2 j}^{2}, v_{2 j+1}^{2}\right) \vee \max _{A_{j}}\left(w_{j}^{2}(a)\right)>\max \left(v_{2 j-1}^{2}, v_{2 j}^{2}, v_{2 j+1}^{2}\right) .
$$


The approximation $w_{j}^{2}(a)$ in element $A_{j}$ is then given by the piecewise linear function (2.18). The total variation of $w_{j}^{2}(a)$ in element $A_{j}$ is then according to Definition 2.1

$$
\begin{aligned}
\operatorname{TV}\left(w_{j}^{2}(a)\right) & =\int_{A_{j}}\left|\frac{\partial w_{j}^{2}}{\partial a}\right| \mathrm{d} a=\left|v_{2 j}^{2}-v_{2 j-1}^{2}\right|+\left|v_{2 j+1}^{2}-v_{2 j}^{2}\right| \\
& =\operatorname{TV}\left(v_{2 j-1}^{2}, v_{2 j}^{2}, v_{2 j+1}^{2}\right) .
\end{aligned}
$$

In case (ii) the quadratic approximation $w_{j}^{2}(a)$ has its extrema in element $A_{j}$ in quadrature points

$$
\min _{A_{j}}\left(w_{j}^{2}(a)\right)=\min \left(v_{2 j-1}^{2}, v_{2 j}^{2}, v_{2 j+1}^{2}\right) \wedge \max _{A_{j}}\left(w_{j}^{2}(a)\right)=\max \left(v_{2 j-1}^{2}, v_{2 j}^{2}, v_{2 j+1}^{2}\right) .
$$

The total variation of $w_{j}^{2}(a)$ in element $A_{j}$ is then

$$
\begin{aligned}
\operatorname{TV}\left(w_{j}^{2}(a)\right) & =\int_{A_{j}}\left|\frac{\partial w_{j}^{2}}{\partial a}\right| \mathrm{d} a=\left|v_{2 j}^{2}-v_{2 j-1}^{2}\right|+\left|v_{2 j+1}^{2}-v_{2 j}^{2}\right| \\
& =\operatorname{TV}\left(v_{2 j-1}^{2}, v_{2 j}^{2}, v_{2 j+1}^{2}\right),
\end{aligned}
$$

which is equal to the result of case (i). For the interpolation $w^{2}(a)$ of the samples $\mathbf{v}^{2}$ over all $n_{\mathrm{e}}$ elements then holds

$$
\operatorname{TV}\left(w^{2}\right)=\sum_{j=1}^{n_{\mathrm{e}}} \operatorname{TV}\left(w_{j}^{2}\right)=\sum_{j=1}^{n_{\mathrm{e}}} \operatorname{TV}\left(v_{2 j-1}^{2}, v_{2 j}^{2}, v_{2 j+1}^{2}\right)=\operatorname{TV}\left(\mathbf{v}^{2}\right) .
$$

Since (2.22) holds for all $\mathbf{v}^{2}$, interpolation method $h^{2}$ is TVC according to Definition 2.3. Lemma 2.1 gives

$$
\operatorname{TV}\left(w^{2}\right)=\operatorname{TV}\left(\mathbf{v}^{2}\right) \leq \operatorname{TV}(u) .
$$

Since (2.23) holds for all $u(a)$, uncertainty quantification method $l^{2}$ is TVD according to Definition 2.2.

Similarly, it can be proven that zero degree Newton-Cotes quadrature in simplex elements is also a TVD uncertainty quantification method for random parameter $a(\omega)$.

\subsubsection{Multi-dimensional probability space}

Consider an uncertainty quantification problem with an arbitrary number of $n_{\mathrm{a}}$ random input parameters $\mathbf{a}(\omega)=\left\{a_{1}(\omega), \cdots, a_{n_{\mathrm{a}}}(\omega)\right\}$ on a bounded connected domain $\mathbf{a} \in A$. In this section it is argued that Stochastic Finite Elements with Newton-Cotes quadrature in simplex elements is also a TVD uncertainty quantification method in the resulting multidimensional probability space for first degree Newton-Cotes. 
Also for an arbitrary number of random parameters $\mathbf{a}(\omega)$ holds that a sampling method $g$ is TVD, since the resulting set of samples $\mathbf{v}$ cannot result in larger total variation than response surface $u(\mathbf{a})$.

Uncertainty quantification method $l^{1}$ based on first degree Newton-Cotes quadrature in simplex elements is also TVD in multi-dimensional probability spaces. The linear interpolation $w_{j}^{1}(\mathbf{a})$ of the samples $\mathbf{v}_{j}^{1}$ in the vertices of simplex element $A_{j}$ conserves the total variation of the samples $\mathbf{v}_{j}$ in element $A_{j}$. Since the piecewise linear interpolation $w^{1}(a)$ of the samples $\mathbf{v}^{1}$ is continuous over the element boundaries $\partial A_{j}$, interpolation $w^{1}(a)$ is TVC with respect to the TVD samples $\mathbf{v}$.

Uncertainty quantification method $l^{2}$ based on second degree Newton-Cotes quadrature in simplex elements results for multi-dimensional probability spaces in an approximation $w^{2}(\mathbf{a})$, which is not everywhere continuous on the element boundaries $\partial A_{j}$. Uncertainty quantification method $l^{2}$ is, therefore, not TVD for multi-dimensional probability spaces.

Zero degree Newton-Cotes quadrature in simplex elements also results in an approximation which is discontinuous at the element boundaries $\partial A_{j}$.

\subsection{Extrema diminishing}

Another important property for uncertainty quantification methods is the extrema diminishing concept. This property eliminates the possibility of predicting non-zero probabilities for unphysical outcomes due to overshoots and undershoots near discontinuities. Consider again an uncertainty quantification problem with an arbitrary number of $n_{\mathrm{a}}$ random input parameters $\mathbf{a}(\omega)=\left\{a_{1}(\omega), \cdots, a_{n_{\mathrm{a}}}(\omega)\right\} \in A$. The concepts extrema diminishing and extrema conserving are defined for probability space below in accordance with their definitions in the context of finite volume methods for physical space [13].

Definition 2.4. (Extrema diminishing) A set of samples $\mathbf{v}$ is extrema diminishing (ED) with respect to response surface $u(\mathbf{a})$ if

$$
\min (\mathbf{v}) \geq \min _{A}(u(\mathbf{a})) \wedge \max (\mathbf{v}) \leq \max _{A}(u(\mathbf{a})) .
$$

Sampling method $g$ is ED if the resulting set of samples $\mathbf{v}$ is ED for all $u(\mathbf{a})$. Approximation $w(\mathbf{a})$ of response surface $u(\mathbf{a})$ is ED if

$$
\min _{A}(w(\mathbf{a})) \geq \min _{A}(u(\mathbf{a})) \wedge \max _{A}(w(\mathbf{a})) \leq \max _{A}(u(\mathbf{a})) .
$$

Uncertainty quantification method $l$ is $\mathrm{ED}$ if the resulting approximation $w(\mathbf{a})$ is ED for all $u(\mathbf{a})$.

Definition 2.5. (Extrema conserving) Interpolation $w(\mathbf{a})$ of samples $\mathbf{v}$ is extrema conserving (EC) if

$$
\min _{A}(w(\mathbf{a}))=\min (\mathbf{v}) \wedge \max _{A}(w(\mathbf{a}))=\max (\mathbf{v}) .
$$

Interpolation method $h$ is EC if the resulting interpolation $w(\mathbf{a})$ is EC for all $\mathbf{v}$. 
It is proven below that the Stochastic Finite Elements method with Newton-Cotes quadrature in simplex elements satisfies the definition of an ED uncertainty quantification method.

Lemma 2.2. Sampling method $g$ is ED.

Proof. For the minimum of the samples $\mathbf{v}$ holds

$$
\min (\mathbf{v})=\min _{k}\left(v_{k}\right)=\min _{k}\left(u\left(\mathbf{a}_{k}\right)\right) \geq \min _{A}(u(\mathbf{a})),
$$

and equivalently for the maximum

$$
\max (\mathbf{v}) \leq \max _{A}(u(\mathbf{a})) .
$$

Since (2.27) and (2.28) hold for all $u(\mathbf{a})$, sampling method $g$ is ED according to Definition 2.4 .

Theorem 2.3. Uncertainty quantification method $l^{1}$ based on first degree Newton-Cotes quadrature in simplex elements is ED.

Proof. For the minimum of the linear interpolation $w_{j}^{1}(\mathbf{a})$ of the samples $\mathbf{v}_{j}^{1}$ in the vertices of simplex element $A_{j}$ holds

$$
\min _{A_{j}}\left(w_{j}^{1}(\mathbf{a})\right)=\min \left(\mathbf{v}_{j}^{1}\right) .
$$

For the minimum of the piecewise linear interpolation $w^{1}(\mathbf{a})$ of the samples $\mathbf{v}^{1}$ then holds

$$
\min _{A}\left(w^{1}(\mathbf{a})\right)=\min _{j}\left(\min _{A_{j}}\left(w_{j}^{1}(\mathbf{a})\right)\right)=\min _{j}\left(\min \left(\mathbf{v}_{j}^{1}\right)\right)=\min \left(\mathbf{v}^{1}\right),
$$

and equivalently for the maximum

$$
\max _{A}\left(w^{1}(\mathbf{a})\right)=\max \left(\mathbf{v}^{1}\right) .
$$

Since (2.30) and (2.31) hold for all $\mathbf{v}^{1}$, interpolation method $h^{1}$ is EC according to Definition 2.5. Lemma 2.2 gives

$$
\min _{A}\left(w^{1}(\mathbf{a})\right)=\min \left(\mathbf{v}^{1}\right) \geq \min _{A}(u(\mathbf{a})), \quad \max _{A}\left(w^{1}(\mathbf{a})\right)=\max \left(\mathbf{v}^{1}\right) \leq \max _{A}(u(\mathbf{a})) .
$$

Since (2.32) holds for all $u(\mathbf{a})$, uncertainty quantification method $l^{1}$ is ED according to Definition 2.4.

Theorem 2.4. Uncertainty quantification method $l^{2}$ based on second degree Newton-Cotes quadrature in simplex elements is ED. 
Proof. The two cases (i) and (ii) again have to be considered to prove Theorem 2.4. In case (i) the quadratic approximation $w_{j}^{2}(\mathbf{a})$ in element $A_{j}$ has an extremum other than in a quadrature point $\mathbf{a}_{k}(2.17)$

$$
\min _{A_{j}}\left(w_{j}^{2}(\mathbf{a})\right)<\min \left(\mathbf{v}_{j}^{2}\right) \vee \max _{A_{j}}\left(w_{j}^{2}(\mathbf{a})\right)>\max \left(\mathbf{v}_{j}^{2}\right) .
$$

The approximation $w_{j}^{2}(\mathbf{a})$ in element $A_{j}$ is then given by a piecewise linear interpolation of the samples $\mathbf{v}_{j}^{2}$, for which holds according to (2.30) and (2.31)

$$
\min _{A_{j}}\left(w_{j}^{2}(\mathbf{a})\right)=\min \left(\mathbf{v}_{j}^{2}\right), \quad \max _{A_{j}}\left(w_{j}^{2}(\mathbf{a})\right)=\max \left(\mathbf{v}_{j}^{2}\right)
$$

In case (ii) the quadratic approximation $w^{2}(\mathbf{a})$ in element $A_{j}$ has its extrema in quadrature points

$$
\min _{A_{j}}\left(w_{j}^{2}(\mathbf{a})\right)=\min \left(\mathbf{v}_{j}^{2}\right) \wedge \max _{A_{j}}\left(w_{j}^{2}(\mathbf{a})\right)=\max \left(\mathbf{v}_{j}^{2}\right),
$$

which is equivalent to the result of case (i). For the minimum and maximum of interpolation $w^{2}(\mathbf{a})$ of samples $\mathbf{v}^{2}$ on $A$ then holds

$$
\min _{A}\left(w^{2}(\mathbf{a})\right)=\min \left(\mathbf{v}^{2}\right), \quad \max _{A}\left(w^{2}(\mathbf{a})\right)=\max \left(\mathbf{v}^{2}\right) .
$$

Since (2.36) holds for all $\mathbf{v}^{2}$, interpolation $h^{2}$ is EC according to Definition 2.5. Lemma 2.2 gives

$$
\min _{A}\left(w^{2}(\mathbf{a})\right)=\min \left(\mathbf{v}^{2}\right) \geq \min _{A}(u(\mathbf{a})), \quad \max _{A}\left(w^{2}(\mathbf{a})\right)=\max \left(\mathbf{v}^{2}\right) \leq \max _{A}(u(\mathbf{a})) .
$$

Since (2.37) holds for all $u(\mathbf{a})$, uncertainty quantification method $l^{2}$ is ED according to Definition 2.4.

Similarly, it can be shown that zero degree Newton-Cotes quadrature in simplex elements is also ED.

\section{Unsteady Adaptive Stochastic Finite Elements}

The Unsteady Adaptive Stochastic Finite Elements method based on interpolation of oscillatory samples at constant phase $\phi$ is introduced in Section 3.1. In Section 3.2 it is proven that the method results in a bounded error as function of the phase for periodic responses. It is also shown under which conditions the error is bounded in time. 


\subsection{Interpolation at constant phase}

Assume that solving Eq. (1.1) for realizations of the random parameters $\mathbf{a}_{k}$ results in oscillatory samples $v_{k}(t)=u\left(\mathbf{a}_{k}\right)$, of which the phase $v_{\phi_{k}}(t)=\phi\left(t, \mathbf{a}_{k}\right)$ is a well-defined function of time $t$. In order to interpolate the samples $\mathbf{v}(t)=\left\{v_{1}(t), \cdots, v_{n_{\mathrm{a}}}(t)\right\}$ at constant phase, first, their phase as function of time $\mathbf{v}_{\phi}(t)=\left\{v_{\phi_{1}}(t), \cdots, v_{\phi_{n_{\mathrm{a}}}}(t)\right\}$ is extracted from the deterministic solves $\mathbf{v}(t)$. Second, the time series for the phase $\mathbf{v}_{\phi}(t)$ are used to transform the samples $\mathbf{v}(t)$ to functions of their phase $\hat{\mathbf{v}}\left(\mathbf{v}_{\phi}(t)\right)=\left\{\hat{v}_{1}\left(v_{\phi_{1}}(t)\right), \cdots, \hat{v}_{n_{\mathrm{a}}}\left(v_{\phi_{n_{\mathrm{a}}}}(t)\right)\right\}$ instead of time, see Fig. 2. Third, the transformed samples $\hat{\mathbf{v}}\left(\mathbf{v}_{\phi}(t)\right)$ are interpolated to the function $\hat{w}\left(w_{\phi}(t, \mathbf{a}), \mathbf{a}\right)$. This step involves both the interpolation of the sampled phases $\mathbf{v}_{\phi}(t)$ to the function $w_{\phi}(t, \mathbf{a})=h\left(\mathbf{v}_{\phi}(t)\right)$ and the interpolation of the samples $\hat{\mathbf{v}}(\varphi)$ to the function $\hat{w}(\varphi, \mathbf{a})=h(\hat{\mathbf{v}}(\varphi))$ at constant phase $\phi=\varphi$. Repeating the latter interpolation for all phases $\varphi$ results in the function $\hat{w}(\varphi, \mathbf{a})$. Finally, transforming $\hat{w}(\varphi, \mathbf{a})$ back to $w(t, \mathbf{a})$ using $w_{\phi}(t, \mathbf{a})$ yields an approximation the unknown response surface $u(t, \mathbf{a})$ of the system response as function of time $t$ and the random parameters $\mathbf{a}(\omega)$. The actual sampling and interpolation is performed using the Adaptive Stochastic Finite Elements uncertainty quantification method $l$ based on Newton-Cotes quadrature in simplex elements.

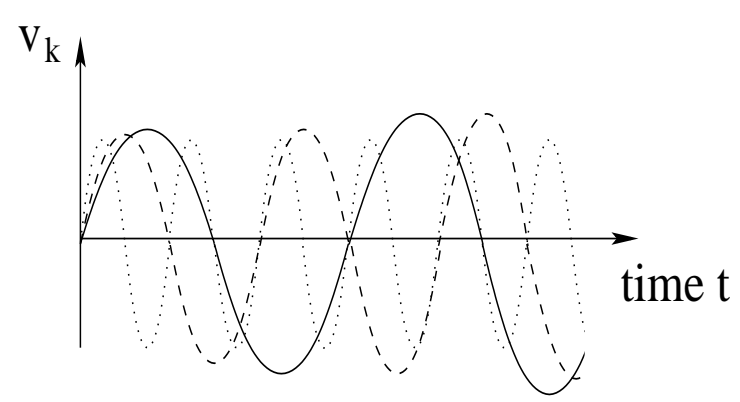

(a) samples $v_{k}(t)$

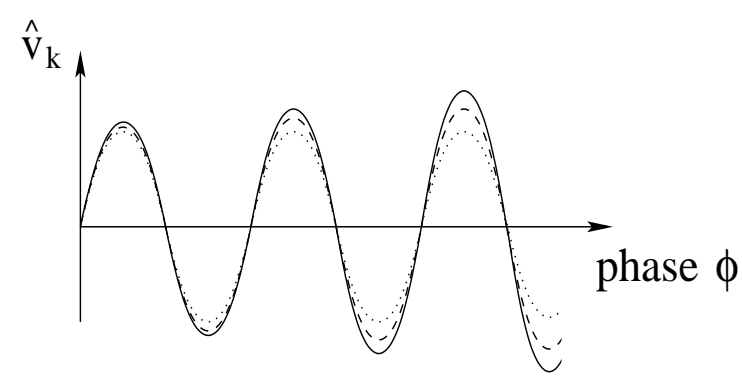

(b) samples $\hat{v}_{k}(\phi)$

Figure 2: Oscillatory samples as function of time and phase.

The phases $\mathbf{v}_{\phi}(t)$ are extracted from the samples based on the local extrema of the time series $\mathbf{v}(t)$. A trial and error procedure identifies a cycle of oscillation based on two or more successive local maxima. The selected cycle is accepted if the maximal error of its extrapolation in time with respect to the actual sample is smaller than a threshold value $\bar{\varepsilon}_{k}$ for at least one additional cycle length. The functions for the phases $\mathbf{v}_{\phi}(t)$ in the whole time domain $T$ are constructed by identifying all successive cycles of $\mathbf{v}(t)$ and extrapolation to $t=0$ and $t=t_{\max }$ before and after the first and last complete cycle, respectively. The phase is normalized to zero at the start of the first cycle and a user defined parameter determines whether the sample is assumed to attain a local extremum at $t=0$. If the phase $\mathbf{v}_{\phi}(t)$ cannot be extracted from one of the samples $v_{k}(t)$ for $k=1, \cdots, n_{\mathrm{s}}$, uncertainty quantification method $l$ is directly applied to the time-dependent samples $\mathbf{v}(t)$. 


\subsection{Bounded error}

It is shown below that the uncertainty quantification interpolation of periodic samples at constant phase results in a bounded error. Let $u(t, \mathbf{a})$ be a periodic response as function of time $t$ for $t \in \mathbb{R}$

$$
u(t+z T(\mathbf{a}), \mathbf{a})=u(t, \mathbf{a}), \quad \text { for all } z \in \mathbb{Z} \text { and } \mathbf{a} \in A,
$$

with $T(\mathbf{a})=1 / f(\mathbf{a})>0$ the period length and $f(\mathbf{a})$ the frequency affected by the random input $\mathbf{a}(\omega)$. The phase $\phi(t, \mathbf{a})$ of the response $u(t, \mathbf{a})$ is given by

$$
\phi(t, \mathbf{a})=\phi_{0}(\mathbf{a})+\frac{t}{T(\mathbf{a})},
$$

with $\phi_{0}(\mathbf{a})=\phi(0, \mathbf{a})$. Consider uncertainty quantification method $l$ which results in an approximation $w(t, \mathbf{a})$ of $u(t, \mathbf{a})$ based on applying interpolation method $h$ at constant phase to $n_{\mathrm{s}}$ samples $\mathbf{v}(t)=\left\{v_{1}(t), \cdots, v_{n_{\mathrm{s}}}\right\}$ for parameter values $\mathbf{a}_{k}$ for $k=1, \cdots, n_{\mathrm{s}}$ resulted from sampling method $g$.

Theorem 3.1. The error $\hat{\varepsilon}(\varphi, \mathbf{a})=\hat{w}(\varphi, \mathbf{a})-\hat{u}(\varphi, \mathbf{a})$ in approximation $\hat{w}(\varphi, \mathbf{a})$ with respect to periodic response surface $\hat{u}(\varphi, \mathbf{a})$ as resulted from uncertainty quantification method l applied at constant phase $\varphi$ is bounded for all $\varphi \in \mathbb{R}$ and $\mathbf{a} \in A$ by $\delta$ for which holds

$$
\hat{\varepsilon}(\varphi, \mathbf{a})<\delta, \quad \text { for all } \varphi \in[0,1] \text { and } \mathbf{a} \in A \text {. }
$$

Proof. Sampling method $g$ results in samples

$$
v_{k}(t)=g_{k}(u(t, \mathbf{a}))=u\left(t, \mathbf{a}_{k}\right),
$$

for $k=1, \cdots, n_{\mathrm{s}}$. The samples $v_{k}(t)$ are periodic signals with period length $v_{T_{k}}=T\left(\mathbf{a}_{k}\right)$, since using (3.1)

$$
\begin{aligned}
v_{k}\left(t+z v_{T_{k}}\right) & =u\left(t+z T\left(\mathbf{a}_{k}\right), \mathbf{a}_{k}\right) \\
& =u\left(t, \mathbf{a}_{k}\right)=v_{k}(t) \quad \text { for all } z \in \mathbb{Z},
\end{aligned}
$$

for $k=1, \cdots, n_{\mathrm{s}}$. The phase $v_{\phi_{k}}(t)=\phi\left(t, \mathbf{a}_{k}\right)$ of the samples $v_{k}(t)$ is then in correspondence with (3.2) given by

$$
v_{\phi_{k}}(t)=v_{\phi_{0_{k}}}+t / v_{T_{k}}, \text { for } k=1, \cdots, n_{\mathrm{s}},
$$

with $v_{\phi_{0_{k}}}=v_{\phi_{k}}(0)$. Scaling the samples $v_{k}(t)$ with their phase $v_{\phi_{k}}(t)$ results in

$$
v_{k}(t)=\hat{v}_{k}\left(v_{\phi_{k}}(t)\right)=\hat{v}_{k}\left(v_{\phi_{0_{k}}}+t / v_{T_{k}}\right),
$$

for $k=1, \cdots, n_{\mathrm{s}}$. Periodicity of $v_{k}(t)$ gives

$$
\begin{aligned}
\hat{v}_{k}\left(v_{\phi_{k}}(t)+z\right) & =\hat{v}_{k}\left(v_{\phi_{0_{k}}}+t / v_{T_{k}}+z\right)=\hat{v}_{k}\left(v_{\phi_{0_{k}}}+\frac{t+z v_{T_{k}}}{v_{T_{k}}}\right) \\
& =v_{k}\left(t+z v_{T_{k}}\right)=v_{k}(t)=\hat{v}_{k}\left(v_{\phi_{k}}(t)\right), \quad \text { for all } z \in \mathbb{Z},
\end{aligned}
$$


for $k=1, \cdots, n_{\mathrm{s}}$. Uncertainty quantification method $l$ results in approximation $\hat{w}(\varphi, \mathbf{a})$ by applying interpolation method $h$ of the samples $\mathbf{v}(t)$ at a constant phase $\varphi$

$$
\hat{w}(\varphi, \mathbf{a})=h(\hat{\mathbf{v}}(\varphi))=h\left(\hat{v}_{1}(\varphi), \cdots, \hat{v}_{n_{\mathrm{s}}}(\varphi)\right) .
$$

The error $\hat{\varepsilon}(\varphi, \mathbf{a})$ as function of phase $\varphi$ in approximation $\hat{w}(\varphi, \mathbf{a})$ with respect to $\hat{u}(\varphi, \mathbf{a})$ is defined as

$$
\hat{\varepsilon}(\varphi, \mathbf{a})=\hat{w}(\varphi, \mathbf{a})-\hat{u}(\varphi, \mathbf{a}),
$$

with

$$
u(t, \mathbf{a})=\hat{u}(\phi(t, \mathbf{a}), \mathbf{a}),
$$

and

$$
\begin{aligned}
\hat{u}(\phi+z, \mathbf{a}) & =\hat{u}\left(\phi_{0}(\mathbf{a})+\frac{t+z T(\mathbf{a})}{T(\mathbf{a})}, \mathbf{a}\right)=u(t+z T(\mathbf{a}), \mathbf{a}) \\
& =u(t, \mathbf{a})=\hat{u}(\phi, \mathbf{a}), \quad \text { for all } z \in \mathbb{Z} \text { and } \mathbf{a} \in A .
\end{aligned}
$$

For error $\hat{\varepsilon}(\varphi, \mathbf{a})$ then holds using (3.8), (3.9), and (3.12)

$$
\begin{aligned}
\hat{\varepsilon}(\varphi+z, \mathbf{a}) & =\hat{w}(\varphi+z, \mathbf{a})-\hat{u}(\varphi+z, \mathbf{a}) \\
& =h\left(\hat{v}_{1}(\varphi+z), \cdots, \hat{v}_{n_{s}}(\varphi+z)\right)-\hat{u}(\varphi+z, \mathbf{a}) \\
& =h\left(\hat{v}_{1}(\varphi), \cdots, \hat{v}_{n_{s}}(\varphi)\right)-\hat{u}(\varphi, \mathbf{a}) \\
& =\hat{w}(\varphi, \mathbf{a})-\hat{u}(\varphi, \mathbf{a})=\hat{\varepsilon}(\varphi, \mathbf{a}), \quad \text { for all } z \in \mathbb{Z} \text { and } \varphi \in \mathbb{R} \text { and } \mathbf{a} \in A .
\end{aligned}
$$

Error $\hat{\varepsilon}(\varphi, \mathbf{a})$ is, therefore, a periodic function of $\varphi$. Define $\delta$ for which holds (3.3)

$$
\hat{\varepsilon}(\varphi, \mathbf{a})<\delta \quad \text { for all } \varphi \in[0,1] \text { and } \mathbf{a} \in A,
$$

then holds

$$
\hat{\varepsilon}(\varphi+z, \mathbf{a})=\hat{\varepsilon}(\varphi, \mathbf{a})<\delta \quad \text { for all } z \in \mathbb{Z} \text { and } \varphi \in[0,1] \text { and } \mathbf{a} \in A,
$$

and

$$
\hat{\varepsilon}(\varphi, \mathbf{a})<\delta \quad \text { for all } \varphi \in \mathbb{R} \text { and } \mathbf{a} \in A,
$$

Error $\hat{\varepsilon}(\varphi, \mathbf{a})$ in approximation $\hat{w}(\varphi, \mathbf{a})$ is, therefore, bounded by $\delta$ for all $\varphi \in \mathbb{R}$ and $\mathbf{a} \in$ A.

Notice that the proof of Theorem 3.1 is independent of uncertainty quantification method $l$, sampling method $g$, and interpolation method $h$.

The bounded error $\hat{\varepsilon}(\varphi, \mathbf{a})$ as function of phase $\varphi$ also results in a bounded error $\varepsilon(t, \mathbf{a})$ in time for the Unsteady Adaptive Stochastic Finite Elements method $l^{1}$ based on first degree Newton-Cotes quadrature, if initial phase $\phi_{0}(\mathbf{a})$ and frequency $f(\mathbf{a})$ depend linearly on a. Let initial phase $\phi_{0}(\mathbf{a})$, therefore, depend linearly on the random parameters a

$$
\phi_{0}(\mathbf{a})=c_{\phi_{0}, 0}+\mathbf{c}_{\phi_{0}, 1} \cdot \mathbf{a} .
$$


where $\cdot$ denotes the vector inner product, with $c_{\phi_{0}, 0}$ constant and $\mathbf{c}_{\phi_{0}, 1}$ a vector containing $n_{\mathrm{a}}$ constants. And let frequency $f(\mathbf{a})$ also depend linearly on $\mathbf{a}(\omega)$

$$
f(\mathbf{a})=c_{\mathrm{f}, 0}+\mathbf{c}_{\mathrm{f}, 1} \cdot \mathbf{a},
$$

with $c_{\mathrm{f}, 0}$ constant and $\mathbf{c}_{\mathrm{f}, 1}$ an $n_{\mathrm{a}}$-dimensional constant vector. Consider uncertainty quantification method $l^{1}$ based on piecewise linear interpolation method $h^{1}$ of samples in the first degree Newton-Cotes quadrature points in the vertices of simplex elements of sampling method $g^{1}$.

Theorem 3.2. The error $\varepsilon(t, \mathbf{a})=w(t, \mathbf{a})-u(t, \mathbf{a})$ in approximation $w(t, \mathbf{a})$ with respect to periodic response surface $u(t, \mathbf{a})$ as resulted from uncertainty quantification method $l^{1}$ applied at constant phase $\varphi$ is bounded for all $t \in \mathbb{R}$ and $\mathbf{a} \in A$ by $\delta$ for which holds (3.3)

$$
\hat{\varepsilon}(\varphi, \mathbf{a})<\delta, \quad \text { for all } \varphi \in[0,1] \text { and } \mathbf{a} \in A,
$$

if initial phase $\phi_{0}(\mathbf{a})$ and frequency $f(\mathbf{a})$ depend linearly on $\mathbf{a}$.

Proof. The phase $\phi(t, \mathbf{a})$ of the periodic response $u(t, \mathbf{a})$ is given by (3.2)

$$
\phi(t, \mathbf{a})=\phi_{0}(\mathbf{a})+\frac{t}{T(\mathbf{a})}=\phi_{0}(\mathbf{a})+f(\mathbf{a}) t .
$$

The linear dependence of $\phi_{0}(\mathbf{a})$ and $f(\mathbf{a})$ on a given by (3.16) and (3.17) results in

$$
\phi(t, \mathbf{a})=c_{\phi_{0}, 0}+c_{\mathrm{f}, 0} t+\left(\mathbf{c}_{\phi_{0}, 1}+\mathbf{c}_{\mathrm{f}, 1} t\right) \cdot \mathbf{a}
$$

The $n_{\mathrm{S}}$ sampled phases $\mathbf{v}_{\phi}^{1}(t)=\left\{v_{\phi_{1}}(t), \cdots, v_{\phi_{n_{\mathrm{S}}}}(t)\right\}$ resulting from sampling method $g^{1}$ are, therefore,

$$
v_{\phi_{k}}^{1}(t)=c_{\phi_{0}, 0}+c_{\mathrm{f}, 0} t+\left(\mathbf{c}_{\phi_{0}, 1}+\mathbf{c}_{\mathrm{f}, 1} t\right) \cdot \mathbf{a}_{k},
$$

for $k=1, \cdots, n_{\mathrm{s}}$. The resulting $w_{\phi}^{1}(t, \mathbf{a})$ of piecewise linear interpolation $h^{1}$ of the samples $\mathbf{v}_{\phi}^{1}(t)$ then exactly reconstructs the function $\phi(t, \mathbf{a})$

$$
w_{\phi}^{1}(t, \mathbf{a})=h^{1}\left(\mathbf{v}_{\phi}^{1}(t)\right)=c_{\phi_{0}, 0}+c_{\mathrm{f}, 0} t+\left(\mathbf{c}_{\phi_{0}, 1}+\mathbf{c}_{\mathrm{f}, 1} t\right) \cdot \mathbf{a}=\phi(t, \mathbf{a}) .
$$

Therefore, error $\hat{\varepsilon}(\phi(t, \mathbf{a}), \mathbf{a})$ in the approximation $\hat{w}^{1}\left(w_{\phi}^{1}(t, \mathbf{a}), \mathbf{a}\right)$ of response $\hat{u}(\phi(t, \mathbf{a}), \mathbf{a})$ becomes

$$
\begin{aligned}
\hat{\varepsilon}(\phi(t, \mathbf{a}), \mathbf{a}) & =\hat{w}^{1}\left(w_{\phi}^{1}(t, \mathbf{a}), \mathbf{a}\right)-\hat{u}(\phi(t, \mathbf{a}), \mathbf{a}) \\
& =\hat{w}^{1}(\phi(t, \mathbf{a}), \mathbf{a})-\hat{u}(\phi(t, \mathbf{a}), \mathbf{a}) \\
& =w^{1}(t, \mathbf{a})-u(t, \mathbf{a}) \\
& =\varepsilon(t, \mathbf{a})<\delta \quad \text { for all } \phi \in \mathbb{R} \text { and } \mathbf{a} \in A,
\end{aligned}
$$

according to Theorem 3.1. Using (3.2) gives

$$
\varepsilon(t, \mathbf{a})<\delta \quad \text { for all } t \in \mathbb{R} \text { and } \mathbf{a} \in A .
$$

This completes the proof of the theorem. 
Unsteady Adaptive Stochastic Finite Elements method $l^{2}$ based on a piecewise quadratic interpolation method $h^{2}$ of samples in the second degree Newton-Cotes quadrature points in simplex elements of sampling method $g^{2}$ consequently gives $\varepsilon(t, \mathbf{a})<\delta$ for all $t \in \mathbb{R}$ and $\mathbf{a} \in A$ up to quadratic dependence of $\phi_{0}(\mathbf{a})$ and $f(\mathbf{a})$ on $\mathbf{a}$.

For periodic responses the interpolation of the samples at constant phase eliminates the effect of the increasing phase differences in time, which usually causes the fast increase of the number of required samples. The error is even bounded in time for periodic problems with an up to quadratic dependence of initial phase $\phi_{0}(\mathbf{a})$ and frequency $f(\mathbf{a})$ on random parameters a. For non-periodic responses the interpolation at constant phase also eliminates the effect of the increasing phase differences on the increase of the number of required samples. The error is for non-periodic responses not bounded in time due to, for example, increasing amplitudes with time. In practice, interpolation of oscillatory samples in time results, however, in an approximately constant accuracy in time with a constant number of samples for periodic and non-periodic responses of which the phase is well-defined.

\section{Numerical results}

The developed uncertainty quantification methods are applied to transonic airfoil flows. Adaptive Stochastic Finite Elements with Newton-Cotes quadrature in simplex elements is applied to a steady transonic airfoil flow in Section 4.1. A transonic airfoil flutter problem is analyzed in Section 4.2 using Unsteady Adaptive Stochastic Finite Elements with interpolation at constant phase.

\subsection{Steady transonic airfoil flow}

The steady transonic flow over a NACA0012 airfoil is considered with randomness in the angle of attack $\alpha(\omega)$. The randomness around the mean angle of attack $\mu_{\alpha}=2^{\circ}$ is given by a symmetrical beta distribution with $\beta_{1}=\beta_{2}=2$ in domain $\alpha \in\left[1^{\circ}, 3^{\circ}\right]$, which corresponds to an input coefficient of variation of $\mathrm{cv}_{\alpha}=22.4 \%$. Standard atmospheric free stream pressure $p_{\infty}=101300 \mathrm{~Pa}$ and temperature $T_{\infty}=293 \mathrm{~K}$ results for free stream velocity $V_{\infty}=276.27 \mathrm{~m} / \mathrm{s}$ in a Mach number of $M_{\infty}=0.8$. The flow is modeled here by the compressible Euler equations [5] mainly to demonstrate the properties of the uncertainty quantification method. The two-dimensional flow domain is discretized by an unstructured hexahedral mesh of $12 \cdot 10^{3}$ cells, which was selected based on a grid convergence study. The Euler equations are discretized using a second order central finite volume discretization stabilized with artificial dissipation [11]. The steady state solution is obtained by time integration with a CFL number of 1.5.

The deterministic flow for the mean angle of attack $\mu_{\alpha}=2^{\circ}$ is transonic with a shock wave at $70.2 \%$ of the upper surface, as can be identified in the pressure field and the pressure distribution over the airfoil surface in Fig. 3. This shock wave in physical space 


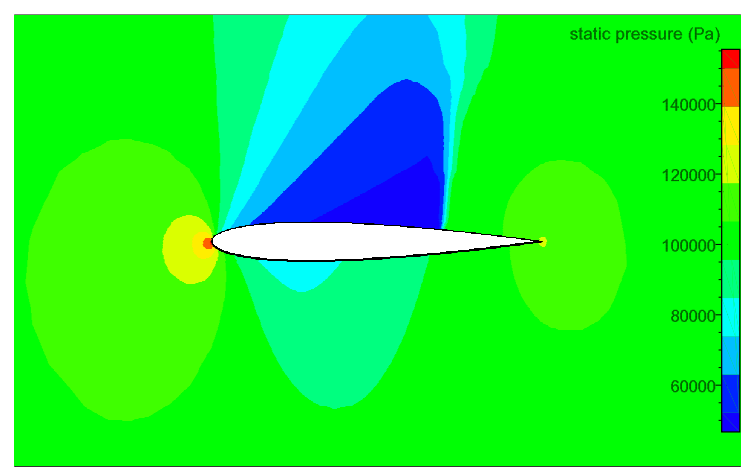

(a) Pressure field

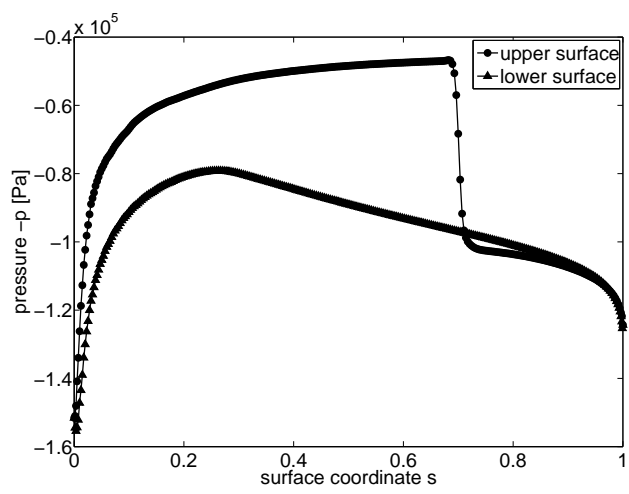

(b) Surface distribution

Figure 3: Deterministic pressure for mean angle of attack $\mu_{\alpha}=2^{\circ}$ for the steady transonic airfoil flow.

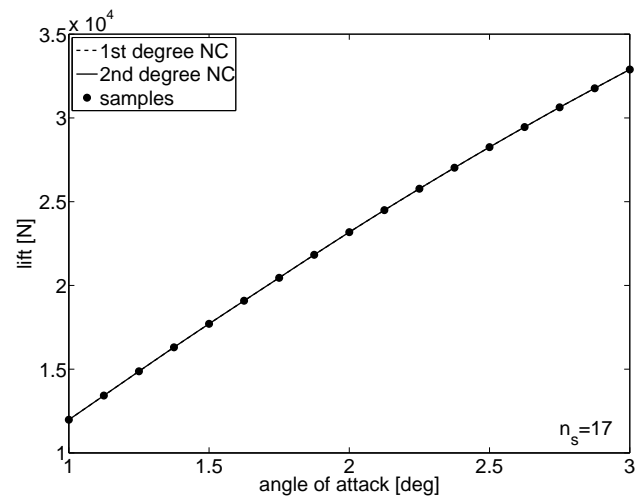

(a) Lift

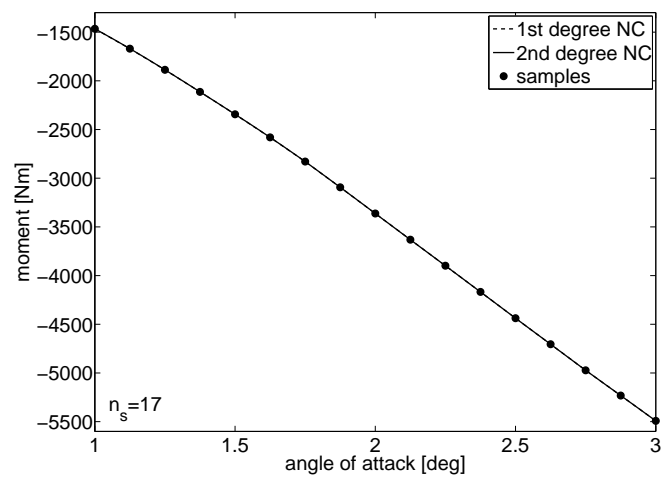

(c) Moment

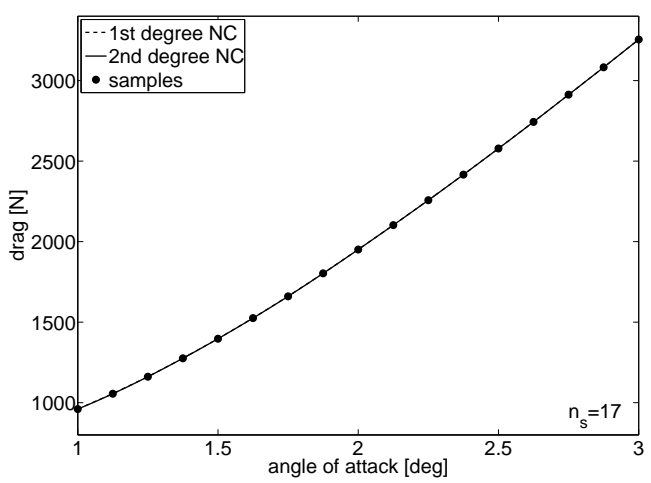

(b) Drag

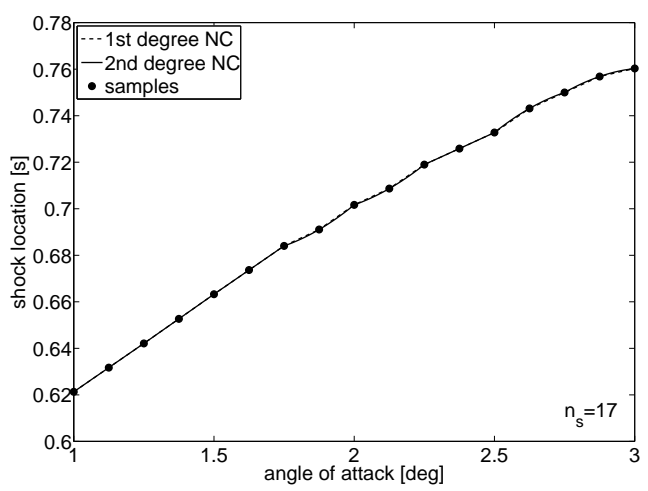

(d) Shock location

Figure 4: Response surfaces of lift, drag, pitching moment, and shock location for the steady transonic airfoil flow with random angle of attack $\alpha(\omega)$. 


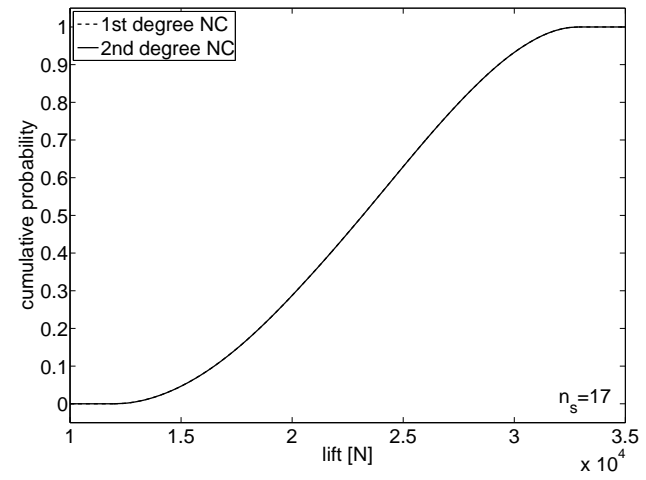

(a) Lift

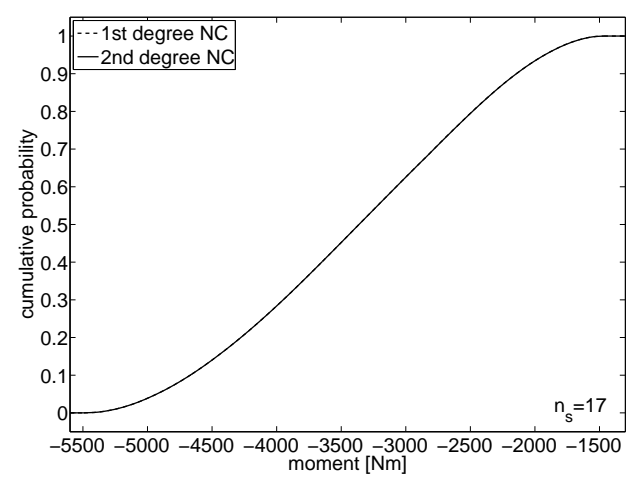

(c) Moment

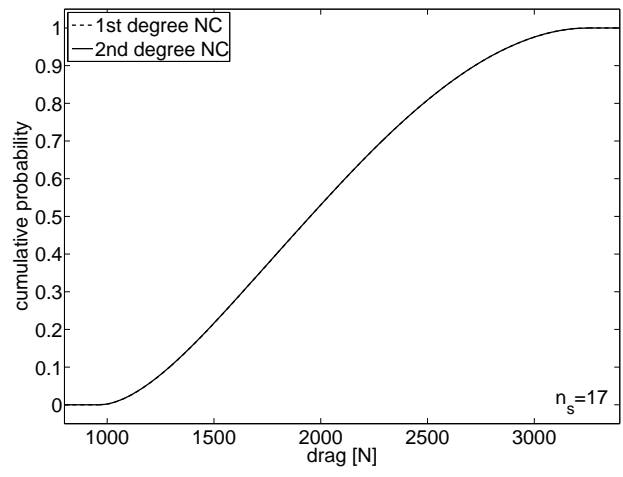

(b) Drag

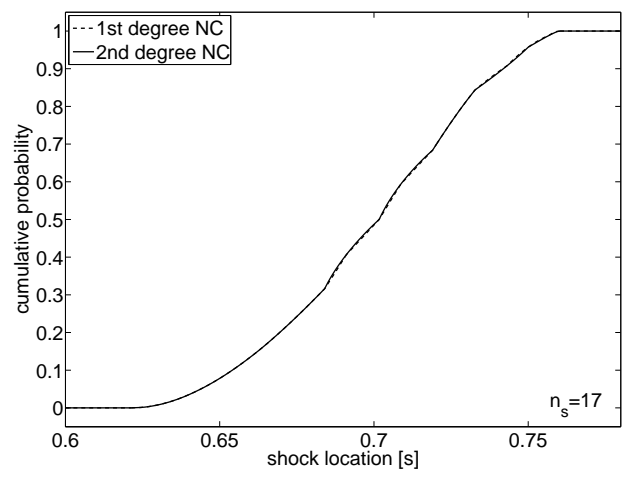

(d) Shock location

Figure 5: Cumulative probability distributions of lift, drag, pitching moment, and shock location for the steady transonic airfoil flow with random angle of attack $\alpha(\omega)$.

results in a discontinuity in probability space. On the lower surface there is also a small supersonic region present.

Adaptive Stochastic Finite Elements with first and second degree Newton-Cotes (NC) quadrature in simplex elements are employed to resolve the response surfaces and probability distributions of the functionals lift, drag, pitching moment, and shock location in Figs. 4 and 5. The first and second degree Newton-Cotes approximations of the response surfaces based on $n_{\mathrm{s}}=17$ samples are in close agreement in Fig. 4 . Both discretizations result in an interpolation that preserves the extrema of the samples. The response surface of the shock location is less smooth than that of the other functionals, because the shock location attains discrete values of the locations of the volume faces on the airfoil surface. Since the multi-element approximations are piecewise continuously differentiable, it is more appropriate to study the variation in the functionals in terms of the resulting cumulative probability distributions in Fig. 5 than in terms of their probability densities. The convergence for the mean and standard deviation of the lift, drag, pitching moment, 
Table 1: Mean and standard deviation of lift $L(\omega)$ for the steady transonic airfoil flow with random angle of attack $\alpha(\omega)$.

\begin{tabular}{|c||c|c|c||c|c|c|}
\hline \multicolumn{1}{|c||}{} & \multicolumn{3}{c||}{ 1st degree NC } & \multicolumn{3}{c|}{ 2nd degree NC } \\
\hline$n_{\mathrm{s}}$ & $n_{\mathrm{e}}$ & mean $\mu_{\mathrm{L}}$ & st.dev. $\sigma_{\mathrm{L}}$ & $n_{\mathrm{e}}$ & mean $\mu_{\mathrm{L}}$ & st.dev. $\sigma_{\mathrm{L}}$ \\
\hline 2 & 1 & $2.244 \cdot 10^{4}$ & $4.676 \cdot 10^{3}$ & - & - & - \\
3 & 2 & $2.290 \cdot 10^{4}$ & $4.679 \cdot 10^{3}$ & 1 & $2.303 \cdot 10^{4}$ & $4.679 \cdot 10^{3}$ \\
5 & 4 & $2.299 \cdot 10^{4}$ & $4.703 \cdot 10^{3}$ & 2 & $2.302 \cdot 10^{4}$ & $4.710 \cdot 10^{3}$ \\
9 & 8 & $2.301 \cdot 10^{4}$ & $4.711 \cdot 10^{3}$ & 4 & $2.302 \cdot 10^{4}$ & $4.714 \cdot 10^{3}$ \\
17 & 16 & $2.302 \cdot 10^{4}$ & $4.712 \cdot 10^{3}$ & 8 & $2.302 \cdot 10^{4}$ & $4.712 \cdot 10^{3}$ \\
\hline
\end{tabular}

Table 2: Mean and standard deviation of drag $D(\omega)$ for the steady transonic airfoil flow with random angle of attack $\alpha(\omega)$.

\begin{tabular}{|c||c|c|c||c|c|c|}
\hline \multicolumn{1}{|c||}{} & \multicolumn{3}{c||}{ 1st degree NC } & \multicolumn{3}{c|}{ 2nd degree NC } \\
\hline$n_{\mathrm{S}}$ & $n_{\mathrm{e}}$ & mean $\mu_{\mathrm{D}}$ & st.dev. $\sigma_{\mathrm{D}}$ & $n_{\mathrm{e}}$ & mean $\mu_{\mathrm{D}}$ & st.dev. $\sigma_{\mathrm{D}}$ \\
\hline 2 & 1 & $2.108 \cdot 10^{3}$ & $5.132 \cdot 10^{2}$ & - & - & - \\
3 & 2 & $2.010 \cdot 10^{3}$ & $5.146 \cdot 10^{2}$ & 1 & $1.982 \cdot 10^{3}$ & $5.143 \cdot 10^{2}$ \\
5 & 4 & $1.988 \cdot 10^{3}$ & $5.231 \cdot 10^{2}$ & 2 & $1.981 \cdot 10^{3}$ & $5.256 \cdot 10^{2}$ \\
9 & 8 & $1.982 \cdot 10^{3}$ & $5.254 \cdot 10^{2}$ & 4 & $1.981 \cdot 10^{3}$ & $5.261 \cdot 10^{2}$ \\
17 & 16 & $1.981 \cdot 10^{3}$ & $5.258 \cdot 10^{2}$ & 8 & $1.981 \cdot 10^{3}$ & $5.259 \cdot 10^{2}$ \\
\hline
\end{tabular}

Table 3: Mean and standard deviation of pitching moment $M(\omega)$ for the steady transonic airfoil flow with random angle of attack $\alpha(\omega)$.

\begin{tabular}{|c||c|c|c||c|c|c|}
\hline \multicolumn{1}{|c||}{} & \multicolumn{3}{c||}{ 1st degree NC } & \multicolumn{3}{c|}{ 2nd degree NC } \\
\hline$n_{\mathrm{S}}$ & $n_{\mathrm{e}}$ & mean $\mu_{\mathrm{M}}$ & st.dev. $\sigma_{\mathrm{M}}$ & $n_{\mathrm{e}}$ & mean $\mu_{\mathrm{M}}$ & st.dev. $\sigma_{\mathrm{M}}$ \\
\hline 2 & 1 & $-3.479 \cdot 10^{3}$ & $9.003 \cdot 10^{2}$ & - & - & - \\
3 & 2 & $-3.406 \cdot 10^{3}$ & $9.008 \cdot 10^{2}$ & 1 & $-3.386 \cdot 10^{3}$ & $9.007 \cdot 10^{2}$ \\
5 & 4 & $-3.390 \cdot 10^{3}$ & $9.219 \cdot 10^{2}$ & 2 & $-3.385 \cdot 10^{3}$ & $9.279 \cdot 10^{2}$ \\
9 & 8 & $-3.385 \cdot 10^{3}$ & $9.275 \cdot 10^{2}$ & 4 & $-3.383 \cdot 10^{3}$ & $9.292 \cdot 10^{2}$ \\
17 & 16 & $-3.384 \cdot 10^{3}$ & $9.282 \cdot 10^{2}$ & 8 & $-3.384 \cdot 10^{3}$ & $9.285 \cdot 10^{2}$ \\
\hline
\end{tabular}

Table 4: Mean and standard deviation of shock location $s_{\text {shock }}(\omega)$ for the steady transonic airfoil flow with random angle of attack $\alpha(\omega)$.

\begin{tabular}{|c||c|c|c||c|c|c|}
\hline \multicolumn{1}{|c||}{} & \multicolumn{3}{c||}{ 1st degree NC } & \multicolumn{3}{c|}{ 2nd degree NC } \\
\hline$n_{\mathrm{s}}$ & $n_{\mathrm{e}}$ & mean $\mu_{\mathrm{s}}$ & st.dev. $\sigma_{\mathrm{s}}$ & $n_{\mathrm{e}}$ & mean $\mu_{\mathrm{s}}$ & st.dev. $\sigma_{\mathrm{s}}$ \\
\hline 2 & 1 & $6.908 \cdot 10^{-1}$ & $3.108 \cdot 10^{-2}$ & - & - & - \\
3 & 2 & $6.976 \cdot 10^{-1}$ & $3.120 \cdot 10^{-2}$ & 1 & $6.995 \cdot 10^{-1}$ & $3.117 \cdot 10^{-2}$ \\
5 & 4 & $6.985 \cdot 10^{-1}$ & $3.118 \cdot 10^{-2}$ & 2 & $6.988 \cdot 10^{-1}$ & $3.118 \cdot 10^{-2}$ \\
9 & 8 & $6.994 \cdot 10^{-1}$ & $3.166 \cdot 10^{-2}$ & 4 & $6.997 \cdot 10^{-1}$ & $3.181 \cdot 10^{-2}$ \\
17 & 16 & $6.992 \cdot 10^{-1}$ & $3.184 \cdot 10^{-2}$ & 8 & $6.991 \cdot 10^{-1}$ & $3.190 \cdot 10^{-2}$ \\
\hline
\end{tabular}

and shock location given in Tables 1 to 4 shows a higher accuracy for second degree Newton-Cotes quadrature compared to first degree quadrature especially for the mean. The standard deviation ranges from $4.6 \%$ of the mean for the shock location to $27.4 \%$ for the pitching moment. 
The effect of the random $\alpha(\omega)$ on the surface pressure distribution in terms of the mean and the $99 \%$ uncertainty interval is given in Fig. 6 for the more accurate second degree Newton-Cotes quadrature. The discretization based on $n_{\mathrm{s}}=17$ samples and $n_{\mathrm{e}}=8$ elements in Fig. 6a shows that the shock wave on the upper surface is smeared out in the mean sense compared to the deterministic case of Fig. 3b. The uncertainty interval in the shock region indicates that $\alpha(\omega)$ has more effect on the shock wave location than on the shock wave strength. This Euler computation also indicates a significantly larger uncertainty interval upstream of the shock than downstream of the shock. The $99 \%$ uncertainty interval falls within the range of the sampled minimum and maximum given by the dotted line in Fig. 6a, which demonstrates the extrema diminishing property of the method. The result for the pressure distribution on the lower surface of Fig. $6 \mathrm{~b}$ predicts a shock wave only for a fraction of the realizations. Discretizations with $n_{\mathrm{s}}=\{3,5,9\}$ show in Fig. $6 c$ convergence of a staircase approximation of the mean and the uncertainty interval to a smooth behavior. Uniform stochastic grid refinement is used here in the examples.

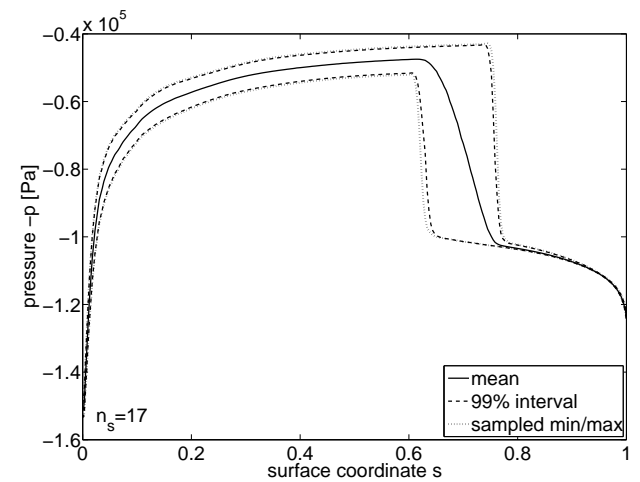

(a) Upper surface

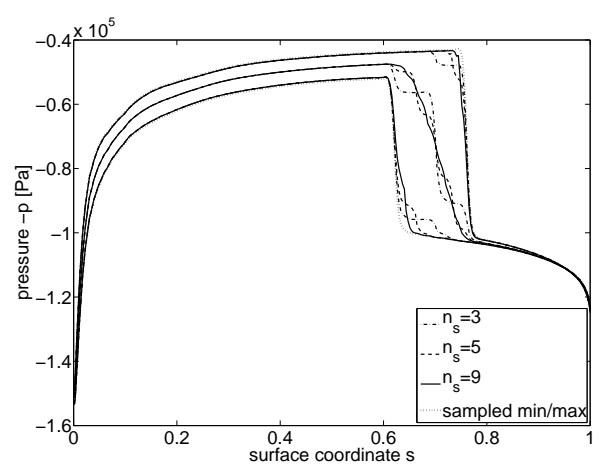

(c) Convergence

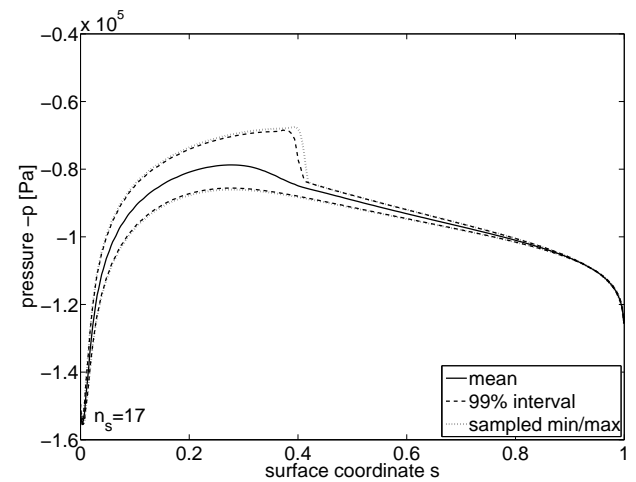

(b) Lower surface

Figure 6: Mean surface pressure and 99\% uncertainty interval of second degree Newton-Cotes quadrature for the steady transonic airfoil flow with random angle of attack $\alpha(\omega)$.

Fig. 7 shows an approximation of the mean and standard deviation of the pressure field relative to the airfoil. In the mean pressure field the smearing of the shock wave 


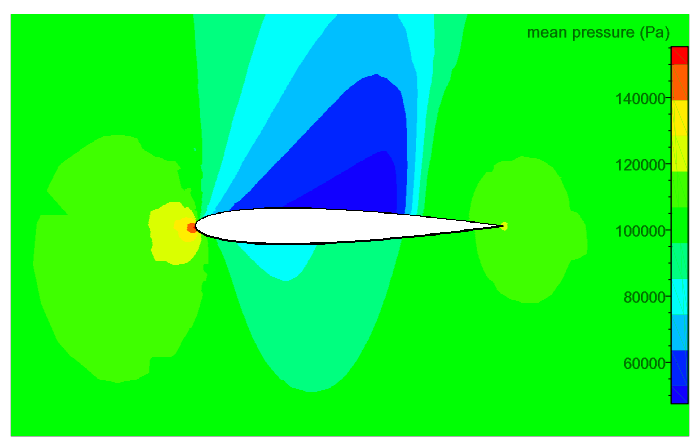

(a) Mean

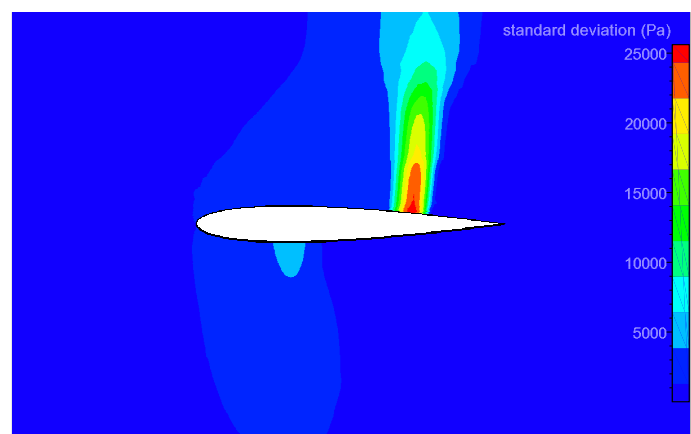

(b) Standard deviation

Figure 7: Mean and standard deviation of the pressure field for second degree Newton-Cotes quadrature with $n_{\mathrm{S}}=17$ for the steady transonic airfoil flow with random angle of attack $\alpha(\omega)$.

can again be identified. The standard deviation field shows that standard deviation is produced in the shock region with a maximum coefficient of variation of $\mathrm{cv}_{\mathrm{p}}=37.1 \%$ at $68.9 \%$ of the upper surface. This corresponds to a maximum amplification of input randomness $\alpha(\omega)$ by $65.6 \%$.

\subsection{Transonic airfoil flutter}

The combined effect of independent randomness in the ratio of natural frequencies $\bar{\omega}(\omega)$ and the free stream velocity $U_{\infty}(\omega)$ on the post-flutter behavior of an elastically mounted airfoil is analyzed. The structural model of the pitch-plunge airfoil with cubic nonlinear spring stiffness is given by $[7,14]$ :

$$
\begin{aligned}
& \xi^{\prime \prime}+x_{\alpha} \alpha^{\prime \prime}+\left(\frac{\bar{\omega}}{U^{*}}\right)^{2}\left(\xi+\beta_{\xi} \xi^{3}\right)=-\frac{1}{\pi \mu} C_{1}(\tau), \\
& \frac{x_{\alpha}}{r_{\alpha}^{2}} \xi^{\prime \prime}+\alpha^{\prime \prime}+\frac{1}{U^{* 2}}\left(\alpha+\beta_{\alpha} \alpha^{3}\right)=\frac{2}{\pi \mu r_{\alpha}^{2}} C_{\mathrm{m}}(\tau),
\end{aligned}
$$

where $\beta_{\xi}=0 \mathrm{~m}^{-2}$ and $\beta_{\alpha}=300 \mathrm{rad}^{-2}$ are the cubic spring parameters, $\xi(\tau)=h / b$ is the nondimensional plunge displacement of the elastic axis, see Fig. $8, \alpha(\tau)$ is the pitch angle, and $(')$ denotes differentiation with respect to non-dimensional time $\tau=U t / b$, with halfchord length $b=c / 2=0.5 \mathrm{~m}$. The radius of gyration around the elastic axis is $r_{\alpha} b=0.25 \mathrm{~m}$, bifurcation parameter $U^{*}$ is defined as $U^{*}=U /\left(b \omega_{\alpha}\right)$, and the airfoil-air mass ratio is $\mu=m / \pi \rho_{\infty} b^{2}=100$, with $m$ the airfoil mass. The elastic axis is located at a distance $a_{\mathrm{h}} b=$ $-0.25 \mathrm{~m}$ from the mid-chord position and the mass center is located at a distance $x_{\alpha} b=$ $0.125 \mathrm{~m}$ from the elastic axis. The ratio of natural frequencies is defined as $\bar{\omega}(\omega)=\omega_{\xi} / \omega_{\alpha}$, with $\omega_{\xi}$ and $\omega_{\alpha}$ the natural frequencies of the airfoil in pitch and plunge, respectively. The randomness in $\bar{\omega}(\omega)$ is described by a uniform distribution around mean value $\mu_{\bar{\omega}}=0.25$ with a coefficient of variation of $10 \%$. The free stream velocity $U_{\infty}(\omega)$ is subject to a 


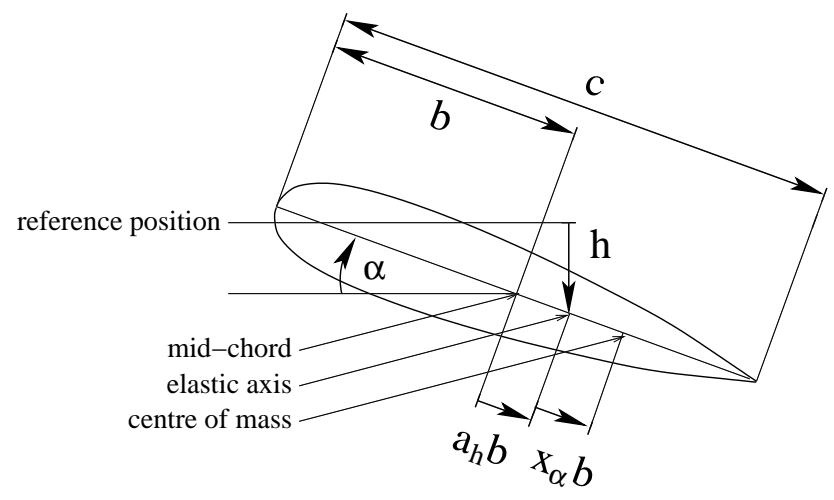

Figure 8: The elastically mounted pitch-plunge airfoil model.

symmetric unimodal beta distribution with $\beta_{1}=\beta_{2}=2$ with a coefficient of variation of $1 \%$ around mean $\mu_{\mathrm{U}_{\infty}}=276.27 \mathrm{~m} / \mathrm{s}$, which corresponds to $M_{\infty}=0.8$.

The non-dimensional aerodynamic lift and moment coefficients, $C_{1}(\tau)$ and $C_{\mathrm{m}}(\tau)$, are determined by solving the Euler equations as for the steady transonic airfoil flow. An Arbitrary Lagrangian-Eulerian formulation is employed to couple the fluid mesh with the movement of the structure. The fluid mesh is deformed using radial basis function interpolation of the boundary displacements [3]. Time integration is performed using the second order BDF-2 method until $t=3$ with time step $\Delta t=0.002$, which was established after a time step refinement study. Initially the airfoil is at rest at a deflection of $\alpha(0)=0.1 \mathrm{deg}$ and $\xi(0)=0$ from its equilibrium position. In order to study the post-bifurcation behavior, the bifurcation parameter $U^{*}$ is fixed at $130 \%$ of the deterministic linear bifurcation point for the mean values of the random parameters. The stochastic behavior of the angle of attack $\alpha(t, \omega)$ is resolved as indicator for the post-flutter airfoil behavior.

The Unsteady Adaptive Stochastic Finite Elements response surface approximation of the angle of attack $\alpha(t, \omega)$ as function of the random parameters $\bar{\omega}(\omega)$ and $U_{\infty}(\omega)$ at $t=\{0.5 ; 1.5 ; 2.5\}$ given in Fig. 9 shows an increasingly oscillatory response surface with time. The $10 \%$ variation in $\bar{\omega}(\omega)$ has a larger effect on the frequency of the response than $U_{\infty}(\omega)$ with $1 \%$ variation. Both parameters have a small effect on the amplitude of the oscillation of $\alpha(t, \omega)$ of approximately $3^{\circ}$. At $t=0.5$ the airfoil exhibits transient behavior from its initial perturbation of $\alpha(0)=0.1^{\circ}$, which is indicated by the smaller amplitude of the response surface variations of approximately $2^{\circ}$. These results are obtained using the time-independent grid in probability space shown in Fig. $9 \mathrm{~d}$ with $n_{\mathrm{s}}=9$ samples, $n_{\mathrm{e}}=2$ elements, and $n_{\mathrm{e}_{\text {sub }}}=4096$ post-processing subelements.

The resulting UASFE approximation of the mean $\mu_{\alpha}(t)$ and standard deviation $\sigma_{\alpha}(t)$ of the angle of attack $\alpha(t, \omega)$ in Fig. 10 shows two frequency signals due to the effect of the two random parameters on the frequency of the response. The mean $\mu_{\alpha}(t)$ exhibits initially an increasing oscillation caused by the deterministic transient of the samples, after which it develops a decaying oscillation due to the effect of the random parameters on the frequency of the response. The large effect of the random parameters on the dy- 


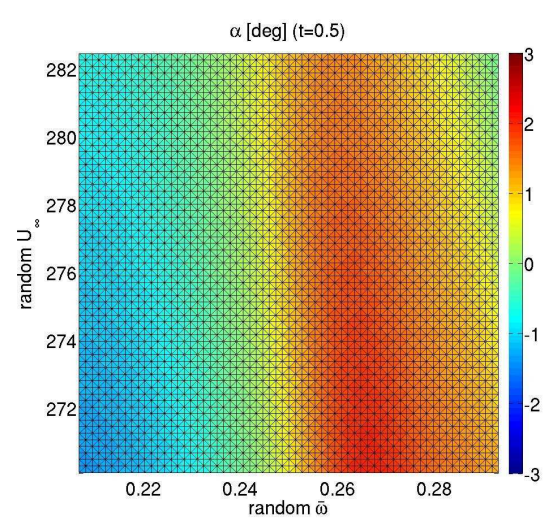

(a) $t=0.5$

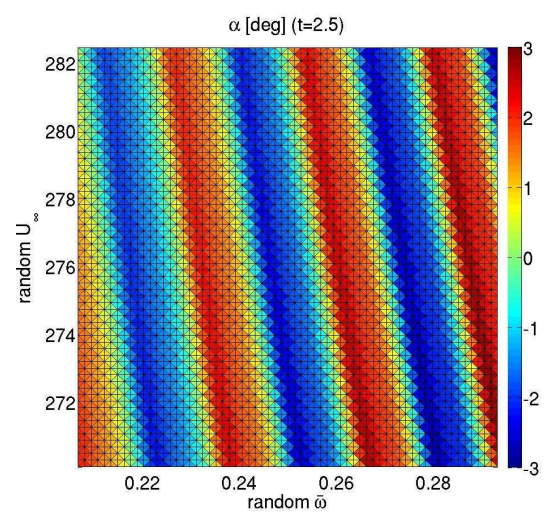

(c) $t=2.5$

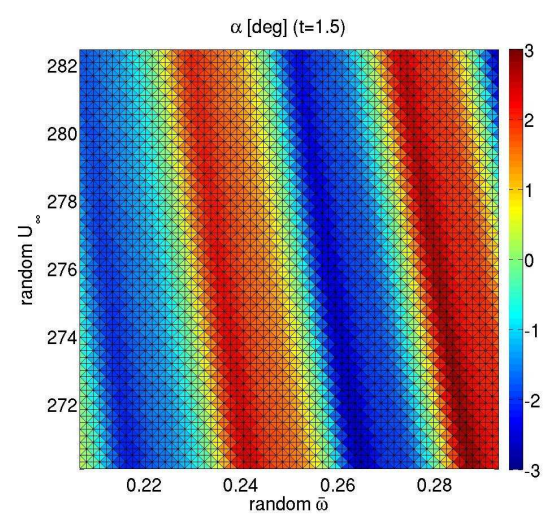

(b) $t=1.5$

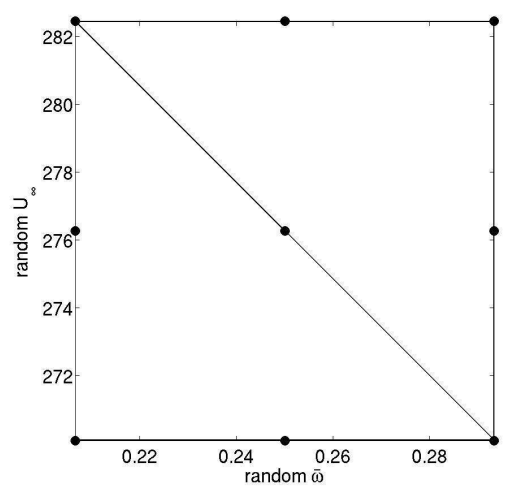

(d) Stochastic grid

Figure 9: Response surface of angle of attack $\alpha(\omega)$ as function of random natural frequency ratio $\bar{\omega}(\omega)$ and free stream velocity $U_{\infty}(\omega)$ for transonic airfoil flutter.

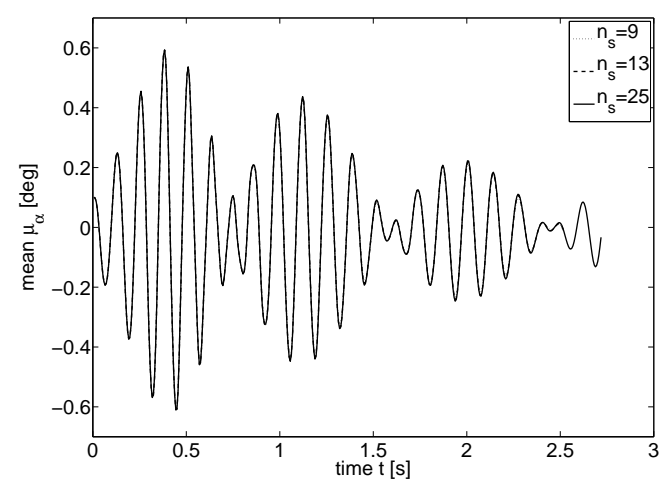

(a) Mean

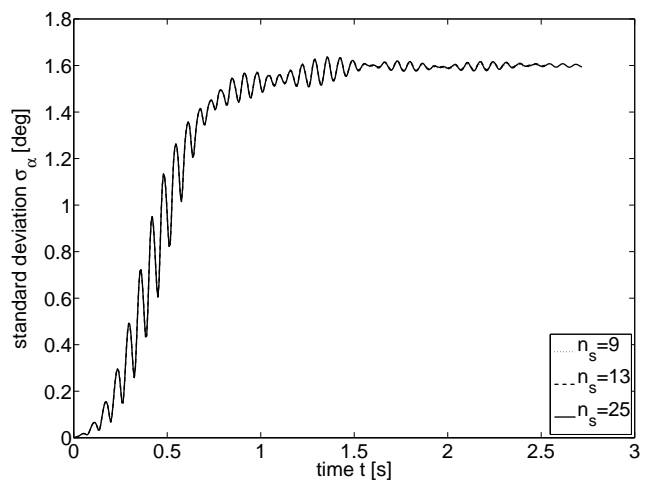

(b) Standard deviation

Figure 10: Mean and standard deviation of angle of attack $\alpha(\omega)$ for transonic airfoil flutter with random natural frequency ratio $\bar{\omega}(\omega)$ and free stream velocity $U_{\infty}(\omega)$. 
Table 5: Convergence measure $\delta_{n_{\mathrm{e}}}$ for mean angle of attack $\alpha(t, \omega)$ for transonic airfoil flutter with random natural frequency ratio $\bar{\omega}(\omega)$ and free stream velocity $U_{\infty}(\omega)$.

\begin{tabular}{|c|c||c|c|c|c|c|}
\hline$n_{\mathrm{s}}$ & $n_{\mathrm{e}}$ & $t=0.5$ & $t=1.0$ & $t=1.5$ & $t=2.0$ & $t=2.5$ \\
\hline 13 & 4 & $0.640 \cdot 10^{-3}$ & $3.712 \cdot 10^{-3}$ & $4.426 \cdot 10^{-3}$ & $7.207 \cdot 10^{-3}$ & $4.331 \cdot 10^{-3}$ \\
25 & 8 & $0.268 \cdot 10^{-3}$ & $2.455 \cdot 10^{-3}$ & $3.138 \cdot 10^{-3}$ & $4.422 \cdot 10^{-3}$ & $2.684 \cdot 10^{-3}$ \\
\hline
\end{tabular}

Table 6: Convergence measure $\delta_{n_{\mathrm{e}}}$ for the standard deviation of angle of attack $\alpha(t, \omega)$ for transonic airfoil flutter with random natural frequency ratio $\bar{\omega}(\omega)$ and free stream velocity $U_{\infty}(\omega)$.

\begin{tabular}{|c|c||c|c|c|c|c|}
\hline$n_{\mathrm{s}}$ & $n_{\mathrm{e}}$ & $t=0.5$ & $t=1.0$ & $t=1.5$ & $t=2.0$ & $t=2.5$ \\
\hline 13 & 4 & $2.943 \cdot 10^{-3}$ & $3.275 \cdot 10^{-3}$ & $7.500 \cdot 10^{-3}$ & $5.378 \cdot 10^{-3}$ & $3.896 \cdot 10^{-3}$ \\
25 & 8 & $0.973 \cdot 10^{-3}$ & $4.388 \cdot 10^{-3}$ & $0.859 \cdot 10^{-3}$ & $2.194 \cdot 10^{-3}$ & $3.344 \cdot 10^{-3}$ \\
\hline
\end{tabular}

namical system is illustrated by the fast initial increase of the standard deviation $\sigma_{\alpha}(t)$ from its deterministic initial condition. Although the deterministic post-flutter behavior is highly unsteady, the stochastic response reaches a steady asymptotic behavior with a standard deviation of $\sigma_{\alpha}=1.6^{\circ}$, which is a factor 16 larger than the initial angle of attack $\alpha(0)=0.1^{\circ}$. The discretizations with $n_{\mathrm{s}}=\{9,13,25\}$ samples and $n_{\mathrm{e}}=\{2,4,8\}$ uniformly refined elements, respectively, indicate that the results are uniformly converged in time. The approximation with $n_{\mathrm{s}}=25$ is converged up to $\delta_{n_{\mathrm{e}}}=6.2 \cdot 10^{-3}$, where $\delta_{n_{\mathrm{e}}}$ is defined by (2.5). The local convergence for $\mu_{\alpha}(t)$ and $\sigma_{\alpha}(t)$ at $t=\{0.5 ; 1.0 ; 1.5 ; 2.0 ; 2.5\}$ given in Tables 5 and 6 for $n_{\mathrm{s}}=\{13,25\}$ shows no clear increase of convergence measure $\delta$ with time. This illustrates that the convergence and the accuracy of the UASFE approximation are in practice constant in time.

\section{Conclusions}

A robust and efficient Unsteady Adaptive Stochastic Finite Elements (UASFE) method is developed for uncertainty quantification in time-dependent simulations. The underlying Adaptive Stochastic Finite Elements (ASFE) discretization based on Newton-Cotes quadrature in simplex elements is extrema diminishing (ED) in probability space. The method is also total variation diminishing (TVD) in probability space for one random parameter and for multiple random parameters for first degree Newton-Cotes quadrature. These properties eliminate the possibility of predicting non-zero probabilities for unphysical outcomes due to overshoots and undershoots at discontinuities. The interpolation of the oscillatory samples at constant phase in the UASFE method results in a bounded error as function of the phase for periodic responses. The UASFE method also results in a bounded error in time, if the initial phase and the frequency of the response depends linearly or quadratically on the random parameters. In practice this results in a constant uncertainty quantification accuracy in time with a constant number of samples.

The applications to a transonic airfoil flow and a transonic airfoil flutter problem show 
a significant effect of input randomness. In the steady transonic airfoil flow randomness in the angle of attack results in production of standard deviation in the shock region with a maximum coefficient of variation $\mathrm{cv}_{\mathrm{p}}=37.1 \%$ at $68.9 \%$ of the upper surface, which corresponds to an amplification of input randomness by $65.6 \%$. The unsteady transonic airfoil flutter problem shows a steady asymptotic stochastic behavior with a standard deviation of $1.6^{\circ}$, which is a factor 16 larger than the deterministic initial condition.

\section{Acknowledgments}

This research was supported by the Technology Foundation STW, applied science division of NWO and the technology programme of the Ministry of Economic Affairs.

\section{References}

[1] I. Babuška, R. Tempone and G. E. Zouraris, Galerkin finite element approximations of stochastic elliptic partial differential equations, SIAM J. Numer. Anal., 42 (2004), 800-825.

[2] I. Babuška, F. Nobile and R. Tempone, A stochastic collocation method for elliptic partial differential equations with random input data, SIAM J. Numer. Anal., 45 (2007), 1005-1034.

[3] A. de Boer, M. S. van der Schoot and H. Bijl, Mesh deformation based on radial basis function interpolation, Comput. Struct., 85 (2007), 784-795.

[4] F. Casciati and B. Roberts, Mathematical Models for Structural Reliability Analysis, CRC Press, Boca Raton, 1996.

[5] A. J. Chorin and J. E. Marsden, A Mathematical Introduction to Fluid Mechanics, SpringerVerlag, New York, 1979.

[6] M. Deb, I. Babuška and J. Oden, Solution of stochastic partial differential equations using Galerkin finite element techniques, Comput. Methods Appl. Mech. Eng., 190 (2001), 63596372.

[7] Y. Fung, An Introduction to Aeroelasticity, Dover Publications, New York, 1969.

[8] R. G. Ghanem and P. Spanos, Stochastic Finite Elements: A Spectral Approach, SpringerVerlag, New York, 1991.

[9] J. M. Hammersley and D. C. Handscomb, Monte Carlo Methods, Methuen's Monographs on Applied Probability and Statistics, Fletcher \& Son Ltd., Norwich, 1964.

[10] A. Harten, High resolution schemes for hyperbolic conservation laws, J. Comput. Phys., 49 (1983), 357-393.

[11] C. Hirsch, Numerical Computation of Internal and External Flows: Fundamentals of Computational Fluid Dynamics, Elsevier, Amsterdam, 2007.

[12] S. Hosder, R. W. Walters and R. Perez, A non-intrusive polynomial chaos method for uncertainty propagation in CFD simulations, AIAA-2006-891, 44th AIAA Aerospace Sciences Meeting and Exhibit, Reno, Nevada, 2006.

[13] A. Jameson, Positive schemes and shock modelling for compressible flows, Int. J. Num. Meth. Fluids, 20 (1995), 743-776.

[14] B. H. K. Lee, L. Y. Jiang and Y. S. Wong, Flutter of an airfoil with a cubic nonlinear restoring force, AIAA-1998-1725, 39th AIAA/ASME/ASCE/AHS/ ASC Structures, Structural Dynamics, and Materials Conference, Long Beach, CA, 1998. 
[15] O. P. Le Maître, O. M. Knio, H. N. Najm and R. G. Ghanem, Uncertainty propagation using Wiener-Haar expansions, J. Comput. Phys., 197 (2004), 28-57.

[16] O. P. Le Maître, H. N. Najm, R. G. Ghanem and O. M. Knio, Multi-resolution analysis of Wiener-type uncertainty propagation schemes, J. Comput. Phys., 197 (2004), 502-531.

[17] L. Mathelin, M. Y. Hussaini and Th. A. Zang, Stochastic approaches to uncertainty quantification in CFD simulations, Num. Alg., 38 (2005), 209-236.

[18] D. R. Millman and P. I. King, Airfoil pitch-and-plunge bifurcation behavior with Fourier chaos expansions, J. Aircraft, 42 (2005), 376-384.

[19] C. L. Pettit and P. S. Beran, Spectral and multiresolution Wiener expansions of oscillatory stochastic processes, J. Sound Vib., 294 (2006), 752-779.

[20] M. T. Reagan, H. N. Najm, R. G. Ghanem and O. M. Knio, Uncertainty quantification in reacting-flow simulations through non-intrusive spectral projection, Combust. Flame, 132 (2003), 545-555.

[21] A. Sarkar and R. G. Ghanem, Mid-frequency structural dynamics with parameter uncertainty, Comput. Method. Appl. Mech. Eng., 191 (2002), 5499-5513.

[22] M. A. Tatang, Direct incorporation of uncertainty in chemical and environmental engineering systems, Ph.D Thesis, MIT, Cambridge, 1995.

[23] X. Wan and G. E. Karniadakis, An adaptive multi-element generalized polynomial chaos method for stochastic differential equations, J. Comput. Phys., 209 (2005), 617-642.

[24] J. A. S. Witteveen and H. Bijl, A monomial chaos approach for efficient uncertainty quantification in nonlinear problems, SIAM J. Sci. Comput., 30 (2008), 1296-1317.

[25] J. A. S. Witteveen, G. J. A. Loeven, S. Sarkar and H. Bijl, Probabilistic collocation for period-1 limit cycle oscillations, J. Sound Vib., 311 (2008), 421-439.

[26] J. A. S. Witteveen and H. Bijl, An unsteady adaptive stochastic finite elements formulation for rigid-body fluid-structure interaction, Comput. Struct., 86 (2008), 2123-2140.

[27] J. A. S. Witteveen and H. Bijl, An alternative unsteady adaptive stochastic finite elements formulation based on interpolation at constant phase, Comput. Method. Appl. Mech. Eng., 198 (2008), 578-591.

[28] J. A. S. Witteveen, G. J. A. Loeven and H. Bijl, An adaptive stochastic finite elements formulation based on Newton-Cotes quadrature in simplex elements, (2008) submitted.

[29] J. A. S. Witteveen and H. Bijl, Effect of randomness on multi-frequency aeroelastic responses resolved by unsteady adaptive stochastic finite elements, (2008) submitted.

[30] D. Xiu and G. E. Karniadakis, The Wiener-Askey polynomial chaos for stochastic differential equations, SIAM J. Sci. Comput., 24 (2002), 619-644.

[31] D. Xiu and J. S. Hesthaven, High-order collocation methods for differential equations with random inputs, SIAM J. Sci. Comput., 27 (2005), 1118-1139.

[32] D. Xiu, Fast numerical methods for stochastic computations: A review, Commun. Comput. Phys., 5 (2009), 242-272. 